\title{
Diencephalic Size Is Restricted by a Novel Interplay Between GCN5 Acetyltransferase Activity and Retinoic Acid Signaling
}

\author{
이onathan J. Wilde, ${ }^{1,2}$ Julie A. Siegenthaler, ${ }^{2}$ CSharon Y.R. Dent, ${ }^{3}$ and Lee A. Niswander ${ }^{2}$ \\ ${ }^{1}$ Graduate Program in Cell Biology, Stem Cells, and Development, University of Colorado Denver, Aurora, Colorado 80045, ${ }^{2}$ Department of Pediatrics, \\ Section of Developmental Biology, University of Colorado School of Medicine, Anschutz Medical Campus, Aurora, Colorado 80045, and 3'University of Texas \\ M.D. Anderson Cancer Center, Department of Epigenetics and Molecular Carcinogenesis, Smithville, Texas 78957
}

Diencephalic defects underlie an array of neurological diseases. Previous studies have suggested that retinoic acid (RA) signaling is involved in diencephalic development at late stages of embryonic development, but its roles and mechanisms of action during early neural development are still unclear. Here we demonstrate that mice lacking enzymatic activity of the acetyltransferase GCN5 $\left(\left(G c n 5^{\text {hat } h a t}\right)\right)$, which were previously characterized with respect to their exencephalic phenotype, exhibit significant diencephalic expansion, decreased diencephalic RA signaling, and increased diencephalic WNT and SHH signaling. Using a variety of molecular biology techniques in both cultured neuroepithelial cells treated with a GCN5 inhibitor and forebrain tissue from (Gcn5 ${ }^{\text {hathat }}$ ) embryos, we demonstrate that GCN5, RAR $\alpha / \gamma$, and the poorly characterized protein TACC1 form a complex in the nucleus that binds specific retinoic acid response elements in the absence of RA. Furthermore, RA triggers GCN5-mediated acetylation of TACC1, which results in dissociation of TACC1 from retinoic acid response elements and leads to transcriptional activation of RA target genes. Intriguingly, RA signaling defects caused by in vitro inhibition of GCN5 can be rescued through RA-dependent mechanisms that require RAR $\beta$. Last, we demonstrate that the diencephalic expansion and transcriptional defects seen in $\left(G c n 5^{\text {hathat }}\right)$ mutants can be rescued with gestational RA supplementation, supporting a direct link between GCN5, TACC1, and RA signaling in the developing diencephalon. Together, our studies identify a novel, nonhistone substrate for GCN5 whose modification regulates a previously undescribed, tissue-specific mechanism of RA signaling that is required to restrict diencephalic size during early forebrain development.

Key words: diencephalon; GCN5; KAT2A; retinoic acid; TACC1; thalamus

Significance Statement

Changes in diencephalic size and shape, as well as SNPs associated with retinoic acid (RA) signaling-associated genes, have been linked to neuropsychiatric disorders. However, the mechanisms that regulate diencephalic morphogenesis and the involvement of RA signaling in this process are poorly understood. Here we demonstrate a novel role of the acetyltransferase GCN5 in a previously undescribed mechanism of RA signaling in the developing forebrain that is required to maintain the appropriate size of the diencephalon. Together, our experiments identify a novel nonhistone substrate of GCN5, highlight an essential role for both GCN5 and RA signaling in early diencephalic development, and elucidate a novel molecular regulatory mechanism for RA signaling that is specific to the developing forebrain.

\section{Introduction}

Microcephaly disorders have provided significant insights into the mechanisms required for achieving proper size of the mam-

\footnotetext{
Received July 1, 2016; revised Jan. 24, 2017; accepted Jan. 25, 2017.

Author contributions: J.J.W., J.A.S., and L.A.N. designed research; J.J.W. performed research; J.J.W., J.A.S., S.Y.R.D., and L.A.N. analyzed data; J.J.W. and L.A.N. wrote the paper.

J.J.W. was supported by National Institute of Neurological Disorders and Stroke Ruth L. Kirschstein National Research Service Award F31NS087692. L.A.N. was an investigator of the Howard Hughes Medical Institute. We thank Lori Bulwith for assistance with animal care; and our laboratory colleagues for insightful discussions.

The authors declare no competing financial interests.

Correspondence should be addressed to either of the following: Dr. Lee A. Niswander, 12801 E. 17th Avenue, Room L18-12106, Aurora, C0 80045, E-mail: lee.niswander@ucdenver.edu; or Dr. Jonathan J. Wilde, Massachusetts Institute of Technology, 43 Vassar Street, Cambridge, MA 02139. E-mail: wildej@mit.edu.
}

malian brain (Gilmore and Walsh, 2013; Lancaster et al., 2013; Chen et al., 2014). However, much less is known about the mechanisms that are required to restrict brain size during development, despite the fact that overgrowth of the whole brain or regions of the brain is associated with several neurological and developmental disorders (Mirzaa et al., 2013). One of the predominant mitogens in the mammalian brain is the secreted morphogen Sonic Hedgehog (SHH) (Rash and Grove, 2011), which plays a fundamental role in the expansion and specification of the diencephalon during early forebrain development (Himmelstein 
et al., 2010; Rash and Grove, 2011; Epstein, 2012). In mice, expression of $S h h$ in the alar plate is responsible for early proliferation of diencephalic tissue and later specification of thalamic nuclei (Rash and Grove, 2011; Chatterjee et al., 2014). However, this Shh expression domain must be restricted as ectopic expression of Shh in the dorsal forebrain has been shown to result in significant expansion of diencephalic tissue and exencephaly (Epstein et al., 2000). Despite these findings, the regulatory pathways required for shaping the diencephalic Shh expression domain are still not fully understood.

Retinoic acid (RA) signaling plays an important role in both development and maintenance of the CNS (Maden, 2007), but its functions in early development of the forebrain are still disputed. Activation of RA-responsive genes occurs through binding of RA to one of three RA receptors (RARs), $\operatorname{RAR} \alpha, \operatorname{RAR} \beta$, or $\operatorname{RAR} \gamma$, at defined genomic loci called retinoic acid response elements (RAREs). These binding events result in recruitment of transcriptional activators and subsequent transcription of downstream target genes (Al Tanoury et al., 2013). Previous studies have shown that RA transcriptional complexes associate with histone acetyltransferases, such as $\mathrm{CBP} / \mathrm{p} 300$, which function through histone modification (Chakravarti et al., 1996; Hou et al., 2015). Whether acetylation of nonhistone targets is important for RA signaling remains unknown. Furthermore, while RA signaling has been intensely studied, there is still little known about its tissue-specific regulation in the CNS. Published work in avian models has implicated RA signaling in restriction of Shh expression in the developing diencephalon; however, this has not been tested in mammals and its mechanism is still unknown (Chambers et al., 2007).

The acetyltransferase GCN5 is typically associated with transcriptional activation through modification of histone $\mathrm{H} 3$. Here we present evidence for a novel mechanism of RA signaling in the murine diencephalon that involves a chromatin-independent function of GCN5 and is required to limit diencephalic expansion during early development of the CNS. First, we show that mice lacking enzymatic activity of GCN5 ( $\left.\left(G c n 5^{\text {hat } / \text { hat }}\right)\right)$ exhibit significant expansion of diencephalic tissue that causes deformation and compression of the telencephalon. Next, using both in situ hybridization and qRT-PCR, we demonstrate increased $\mathrm{SHH}$ and WNT signaling, which have previously been shown to positively regulate proliferation of the diencephalon. We then use a combination of qRT-PCR and a reporter of RA signaling to demonstrate that diencephalic RA signaling is specifically disrupted in $\left(G c n 5^{\text {hat } / \text { hat }}\right)$ embryos and lies upstream of the changes in $\mathrm{SHH}$ and WNT signaling. Next, using cultured neuroepithelial cells and embryonic tissue, we identify a novel mechanism of RA signaling in the developing forebrain involving GCN5-mediated acetylation of TACC1, which acts as a repressor of RAR $\alpha / \gamma$ dependent RA signaling in the absence of ligand. Last, we show that the RA signaling defects and diencephalic expansion in $\left(G c n 5^{\text {hat } / \text { hat }}\right)$ embryos can be rescued through dietary supplementation of RA through a mechanism requiring RAR $\beta$. Together, our studies identify a novel mechanism of diencephalic size regulation that requires acetylation of TACC1, a previously unidentified substrate of GCN5, for transcriptional activation of diencephalic targets of RA signaling.

\section{Materials and Methods}

Mouse strains and genotyping. All animals were maintained according to protocols approved by the University of Colorado Denver Institutional Animal Care and Use Committee. (Gcn $5^{\text {hat }}$ ) mice were genotyped according to previously published protocols (Bu et al., 2007) and main- tained on a C57BL/6J background (RRID:IMSR-JAX:000664). Gcn5 $5^{\text {hat/+ }}$ RAREhspLacZ mice were generated by crossing Gcn $5^{\text {hat/+ }}$ mice with homozygous $\operatorname{Tg}(R A R E-H s p a 1 b / L a c Z) 12 J$ tr/J mice (RRID:IMSR-JAX: 008477) and were maintained as F1 offspring to avoid disruption of reporter activity by the ( $\left.G c n 5^{\text {hat }}\right)$ C57BL/6J background. For all experiments, wild-type and (Gcn5 $\left.5^{\text {hat } / h a t}\right)$ embryos of comparable age based on somite number were used for comparison. All experiments were performed with embryos of either sex.

Immunohistochemistry and confocal microscopy. Embryos were fixed for $30 \mathrm{~min}$ at room temperature in $4 \%$ PFA and placed in 30\% sucrose overnight at $4^{\circ} \mathrm{C}$ on a nutator. Heads were embedded in OCT Compound (Tissue-Tek) and frozen on dry ice before storage at $-80^{\circ} \mathrm{C}$. The $14 \mu \mathrm{m}$ coronal cryosections were cut on a Leica CM3050S and mounted on Superfrost Plus slides (Fisher). For immunostaining, slides were dried for $30 \mathrm{~min}$ at room temperature, subjected to heat mediated antigen retrieval in sodium citrate buffer ( $10 \mathrm{~mm}$ sodium citrate, $\mathrm{pH} 6.0$ ) for $12 \mathrm{~min}$, blocked for $1 \mathrm{~h}$ at room temperature, and stained with primary antibody overnight at $4{ }^{\circ} \mathrm{C}$. For indicated antibodies, staining after antigen retrieval was performed using a Tyramide Signal Amplification (TSA) kit (Invitrogen) according to the manufacturer's protocols. Primary antibodies used were as follows: rabbit anti-FOXG1 (Abcam catalog \#ab18259 RRID:AB-732415, 1:100), rabbit anti-LEF1 (Cell Signaling Technology catalog \#2230S RRID:AB-823558, 1:500, TSA kit), mouse anti-PAX6 (DSHB catalog \#pax6 RRID:AB-2315070, 1:50), mouse anti-P21 (BD Biosciences catalog \#556431 RRID:AB-396415, 1:1000, TSA kit), mouse anti-MASH1 (BD Biosciences catalog \#556604 RRID:AB-396479, 1:50, TSA kit), and rabbit anti-TBR2 (Abcam catalog \#ab23345 RRID:AB778267, 1:400), mouse anti-NKX2.2 (DSHB catalog \#74.5A5 RRID:AB531794, 1:10), mouse anti-OLIG2 (Millipore catalog \#MABN50 RRID: AB-10807410, 1:200), and mouse anti-PAX7 (DSHB catalog \#PAX7 RRID:AB-2299243, 1:50).

Secondary antibody staining was performed for $1 \mathrm{~h}$ at room temperature, and slides were mounted using Faramount mounting media (Dako). The following secondary antibodies were used at 1:250 in blocking buffer for $1 \mathrm{~h}$ at room temperature: AlexaFluor goat anti-rabbit 488 (Invitrogen catalog \#A-11008 RRID:AB-143165), AlexaFluor goat antimouse 488 (Invitrogen catalog \#A-11001 RRID:AB-141367), AlexaFluor goat anti-rabbit 555 (Invitrogen catalog \#A-21428 RRID:AB-131784), and AlexaFluor goat anti-mouse 555 (Invitrogen catalog \#A-21422 RRID:AB-141822). Imaging was performed on a Zeiss LSM510 Meta laser scanning confocal microscope using Zen software. Scale bars were added using FIJI (Schindelin et al., 2012), and brightness, contrast, and color levels were adjusted using Photoshop CS5 (Adobe Systems).

Whole-mount in situ hybridization. Whole-mount in situ hybridization was performed as previously described (Holmes and Niswander, 2001). The following probes were used: mShh (HindIII, T3; provided by A. Joyner), mGli3 (NcoI, SP6; provided by J. Eggenschwiler), mAxin2 (XbaI, Sp6; provided by M. Buckingham), and mWnt8b (EcoRI, T7; provided by E. Grove).

$q R T-P C R$. RNA was isolated according to the manufacturer's instructions using the High Pure RNA Isolation Kit (Roche), and cDNA was generated using random hexamers and the Transcriptor First Strand cDNA Synthesis Kit (Roche). qPCR was performed on a LightCycler 480 (Roche) using the LightCycler 480 Probes Master reagent (Roche) and Universal Probe Library (Roche) according to the manufacturer's protocol. All primers and probes are available upon request. A combination of Rrn18s and Gusb was used for normalization in each experiment. Data were collected and analyzed with LightCycler 480 Software (Roche, version 1.5.1).

Whole-mount $\beta$-galactosidase assays. E10.5 embryos were washed briefly in PBT (PBS $+0.1 \%$ Tween 20 ) and fixed at room temperature in $4 \%$ PFA for $45 \mathrm{~min}$. Embryos were then washed three times for $15 \mathrm{~min}$ each at room temperature in wash buffer $\left(0.2 \mathrm{~mm} \mathrm{MgCl}_{2}, 0.02 \% \mathrm{NP}-40\right.$, $0.01 \%$ deoxycholate in PBT) before staining overnight at $37^{\circ} \mathrm{C}$ in $\mathrm{X}$-gal staining solution $(1 \mathrm{mg} / \mathrm{ml} \mathrm{X}$-gal, $5 \mathrm{~mm}$ potassium ferrocyanide, $5 \mathrm{~mm}$ potassium ferricyanide in wash buffer). Embryos were then briefly washed in wash buffer and fixed for $30 \mathrm{~min}$ at room temperature before imaging on a Nikon SMZ18 stereomicroscope using Nikon NISElements software (version 4.13). 
Cell culture. NE-4C cells (ATCC catalog \#CRL-2925, RRID:CVCL$\mathrm{B} 063$ ) were grown at $37^{\circ} \mathrm{C}$ and $5 \% \mathrm{CO}_{2}$ in MEM (Invitrogen) supplemented with 5\% FBS, $1 \times$ MEM nonessential amino acids (Invitrogen), and $1 \times$ GlutaMax (Invitrogen). For RA treatments, cells were treated with $0.01-10 \mu \mathrm{M}$ all-trans retinoic acid (Sigma-Aldrich) dissolved in DMSO. DMSO was used as a vehicle control for all experiments. For MB-3 treatments, MB-3 (Sigma-Aldrich) was prepared in sterile $\mathrm{dd}_{2} \mathrm{O}$ at a stock concentration of $100 \mathrm{~mm}$ and used at final concentrations between 100 and $200 \mu \mathrm{M}$.

Western blot imaging. Western blots were visualized on an Odyssey CLx (LI-COR Biosciences) with the following LI-COR secondary antibodies: IRDye 680 goat anti-mouse IgG $(\mathrm{H}+\mathrm{L})$ (LI-COR Biosciences catalog \#926-68070 RRID:AB-10956588), IRDye 680 donkey anti-goat IgG (H+L) (LI-COR Biosciences catalog \#926-68024 RRID:AB10706168), IRDye 680 goat anti-rabbit IgG $(\mathrm{H}+\mathrm{L})$ (LI-COR Biosciences catalog \#926-68021 RRID:AB-10706309), IRDye 800CW goat antimouse IgG $(\mathrm{H}+\mathrm{L})$ (LI-COR Biosciences catalog \#926-32210 RRID:AB621842), and IRDye 800CW goat anti-rabbit IgG (H+L) (LI-COR Biosciences catalog \#926-32211 RRID:AB-621843). Western blots were quantified using Image Studio software (LI-COR Biosciences).

Protein acetylation assay. NE-4C cells treated with vehicle control (DMSO, 1:1000), $100 \mu \mathrm{M}$ MB-3, $0.1 \mu \mathrm{M}$ RA, or $100 \mu \mathrm{M} \mathrm{MB}-3$ and $0.1 \mu \mathrm{M}$ RA were grown for $16 \mathrm{~h}$, and subcellular fractionation was performed using the Nuclear Complex coimmunoprecipitation kit (Clontech). For in vivo protein acetylation assays, E10.5 forebrain tissue was dissected and directly transferred to RIPA buffer. Total protein was isolated via passive lysis at $4^{\circ} \mathrm{C}$ and collected after centrifugation. Indirect immunoprecipitation was performed on $500 \mu \mathrm{g}$ of input using rabbit antiacetylated lysine antibody (Cell Signaling Technology catalog \#9441S RRID: AB-331805, 1:100) or normal rabbit IgG (Millipore catalog \#12-371 RRID:AB-145840, $2 \mu \mathrm{g}$ ) and PureProteome Protein G Magnetic Beads (Millipore) according to the manufacturer's protocol. To quantify acetylated TACC1, Western blotting was performed on IP and input using rabbit anti-TACC1 (Sigma-Aldrich catalog \#SAB4502910 RRID:AB10747337, 1:500) and mouse anti- $\beta$-tubulin (Sigma-Aldrich catalog \#T4026 RRID:AB-477577, 1:2000). Total TACC1 was normalized to $\beta$-tubulin, and acetylated TACC1 was then normalized to total TACC1. Efficiency of fractionation and loading consistency was assayed via Western blot with antibodies directed against RNA polymerase II (Millipore catalog \#05-623 RRID:AB-309852, 1:500) and $\beta$-tubulin.

Coimmunoprecipitation. Freshly dissected embryonic forebrain tissue or NE-4C cells grown for $16 \mathrm{~h}$ after treatment with DMSO, 50 or $100 \mu \mathrm{M}$ MB-3, $0.1 \mu \mathrm{M}$ RA, or 50 or $100 \mu \mathrm{M}$ MB-3 and $0.1 \mu \mathrm{M}$ RA were collected, and nuclear protein was harvested using the Nuclear Complex coimmunoprecipitation kit according to the manufacturer's protocol (Clontech). The $750 \mu \mathrm{g}$ input $(5 \mu \mathrm{g} / \mu \mathrm{l})$ was precleared with $20 \mu \mathrm{l}$ PureProteome Protein G Magnetic Beads (Millipore) before addition of rabbit antiRAR $\alpha$ (Santa Cruz Biotechnology catalog \#sc-551 RRID:AB-2177750, 1:200) or normal rabbit IgG. Samples were rotated overnight at $4^{\circ} \mathrm{C}$ before addition of $50 \mu \mathrm{l}$ washed PureProteome Protein G Magnetic Beads and subsequent incubation at room temperature for $15 \mathrm{~min}$ on a nutator. Beads were then washed 5 times in ice-cold wash buffer before elution in $2 \times$ Laemmli buffer at $95^{\circ} \mathrm{C}$ for $10 \mathrm{~min}$. Protein interactions were assayed via Western blot using the following antibodies: rabbit anti-GCN5L2 (Cell Signaling Technology catalog \#3305S RRID:AB2128281, 1:1000), goat anti-RAR $\alpha$ (Santa Cruz Biotechnology catalog \#sc-15040 RRID:AB-2177747, 1:50), rabbit anti-TACC1 (Sigma-Aldrich catalog \#SAB4502910 RRID:AB-10747337, 1:500), and mouse anti- $\beta$ tubulin (Sigma-Aldrich catalog \#T4026 RRID:AB-477577, 1:2000).

Chromatin immunoprecipitation (ChIP). NE-4C cells were grown for $24 \mathrm{~h}$ under the indicated conditions before proteins were cross-linked to DNA by addition of methanol-free formaldehyde to a final concentration of $0.75 \%$ for $10 \mathrm{~min}$ at room temperature. After quenching of formaldehyde with glycine (final concentration $125 \mathrm{~mm}$ ), cells were washed with ice-cold PBS and protein was harvested via passive lysis with FA lysis buffer (50 mм HEPES-KOH pH 7.5, 140 mм NaCl, 1 mм EDTA pH 8.0, $1 \%$ Triton X-100, 0.1\% sodium deoxycholate, 0.1\% SDS, 1 EDTA-free protease inhibitors). DNA was sheared to $\sim 500 \mathrm{bp}$ fragments using a Bioruptor (Diagenode) at high intensity for $45 \mathrm{~min}$ ( $30 \mathrm{~s}$ on, $30 \mathrm{~s}$ off).
Samples were centrifuged at $8000 \times g$ for $30 \mathrm{~s}$ at $4^{\circ} \mathrm{C}$, and the supernatant was transferred to a new tube. Protein-DNA complexes were incubated with the following antibodies overnight at $4^{\circ} \mathrm{C}$ on a rotator: normal rabbit IgG (Millipore catalog \#12-371 RRID:AB-145840, $2 \mu \mathrm{g}$ ), rabbit anti-GCN5L2 (Cell Signaling Technology catalog \#3305S RRID:AB2128281, 1:200), rabbit anti-RAR $\alpha$ (Santa Cruz Biotechnology catalog \#sc-551 RRID:AB-2177750, 1:200), rabbit anti-TACC1 (Sigma-Aldrich catalog \#SAB4502910 RRID:AB-10747337, 1:100). The $50 \mu$ l ChIP-grade Protein G Magnetic Beads (Cell Signaling Technology) preabsorbed with $3.75 \mu \mathrm{g}$ sheared single-stranded herring sperm DNA was added to each sample and incubated for $2 \mathrm{~h}$ at $4^{\circ} \mathrm{C}$ on a rotator. Beads were collected via magnet and washed 3 times in ice-cold wash buffer $(0.1 \%$ SDS, $1 \%$ Triton X-100, 2 mм EDTA pH 8.0, 150 mм NaCl, 20 mм Tris-HCl pH 8.0 ) before elution in $120 \mu$ l elution buffer ( $1 \%$ SDS, $100 \mathrm{~mm} \mathrm{NaHCO}_{3}$ ) on a Thermomixer (Eppendorf) at $750 \mathrm{rpm}$ and $30^{\circ} \mathrm{C}$ for $15 \mathrm{~min}$. DNA was then purified via QiaQuick PCR Purification Kit (QIAGEN) according to the manufacturer's instructions. qPCR was performed using LightCycler 480 SYBR Green I Master reagent (Roche). Reactions were run on a LightCycler 480 (Roche) and analyzed with LightCycler 480 Software (Roche, version 1.5.1). Target data were first normalized to input levels and then normalized to IgG control. ChIP primer sequences are as follows: Rarb-RARE1-F 5' -CGGAGCAGCTCACTTCCTAC-3' + Rarb-RA RE2-F 5'-CTGGTTGGGTCATTTGAAGG (121 bp product), RarbRARE2-F 5'-ACAGATGGCACTGAGAAGGC-3' + Rarb-RARE2-R 5'-CGAACTCAGATGCACAATGC-3' (120 bp product), and FoxalRARE-F 5'-CTCTGGGACCAAGCTAGAGG + Foxa1-RARE-R 5'CTCATTAGCGTGGCAGATG-3' (137 bp product).

shRNA transfection. shRNAs were transfected using the Xfect Transfection Reagent (Clontech) according to the manufacturer's protocol (7.5 $\mu \mathrm{g}$ plasmid per well of a 6-well plate). Media was changed $4 \mathrm{~h}$ after transfection and cells were grown for $24 \mathrm{~h}$ before being used for experiments. The following Mission shRNA (Sigma) constructs were used: pLKO.1-puro Non-Target shRNA control (SHC002), TRCN0000027121 (Rarb), and TRCN0000027071 (Rarb).

$R A$ agonist experiments. NE- $4 \mathrm{C}$ cells were plated at a density of $1.0 \times$ $10^{5}$ cells/well in individual wells of a 24 -well plate in $500 \mu \mathrm{l}$ culture medium. Plated cells were allowed to grow overnight before addition of DMSO (1:1000), all-trans retinoic acid $(0.1 \mu \mathrm{M}), \mathrm{MB}-3(200 \mu \mathrm{M})$, selective RAR $\alpha$ agonist BMS 753 (Tocris Bioscience; $1 \mu \mathrm{M}$ ), selective RAR $\beta$ agonist CD 2314 (Tocris Bioscience; $1 \mu \mathrm{M}$ ), or selective RAR $\gamma$ agonist CD 437 (Tocris Bioscience; $0.1 \mu \mathrm{M}$ ). Some wells were treated with a combination of drugs, as indicated in Figure 6. Drug treatment was performed for $24 \mathrm{~h}$.

Gestational RA supplementation. Pregnant dams were provided alltrans retinoic acid (Sigma) at $175 \mathrm{mg} / \mathrm{kg}$ food ad libitum beginning at gestational day 8.5. RA was added to $1 \mathrm{ml}$ of corn oil and mixed with $8 \mathrm{~g}$ of Nutra-Gel diet (BioServ, \#S4798-TRAY), which was then provided in place of normal mouse chow. Fresh food and RA were provided each morning for the duration of the diet.

Statistical analysis. All statistical analyses were performed using Prism 6 (GraphPad). For Figures $4 C, 5 D$, and $6 B, E, G$, J, a two-way ANOVA with Tukey's multiple-comparison's test was performed. For all other figures, unpaired $t$ tests were performed. All graphed and numerical data represent mean $\pm \mathrm{SD}$.

\section{Results}

\section{Expansion and mispatterning of the diencephalon in} $\left(G c n 5^{\text {hathat }}\right)$ mutants

GCN5 is an acetyltransferase first identified as a positive regulator of transcription that functions directly through lysine acetylation on histone H3 (Georgakopoulos and Thireos, 1992; Candau et al., 1996; Brand et al., 1999; Grant et al., 1999). It is a member of the SAGA coactivator complex that regulates POLII-mediated transcription (Grant et al., 1999), and it has been shown to acetylate several nonhistone targets (Jacob et al., 2001; Lerin et al., 2006; Conacci-Sorrell et al., 2010; Zelin et al., 2012; L. Li et al., 2015). Gcn5-null mice die early in gestation after failing to specify mesodermal tissues (Xu et al., 2000), but mice engineered to lack 

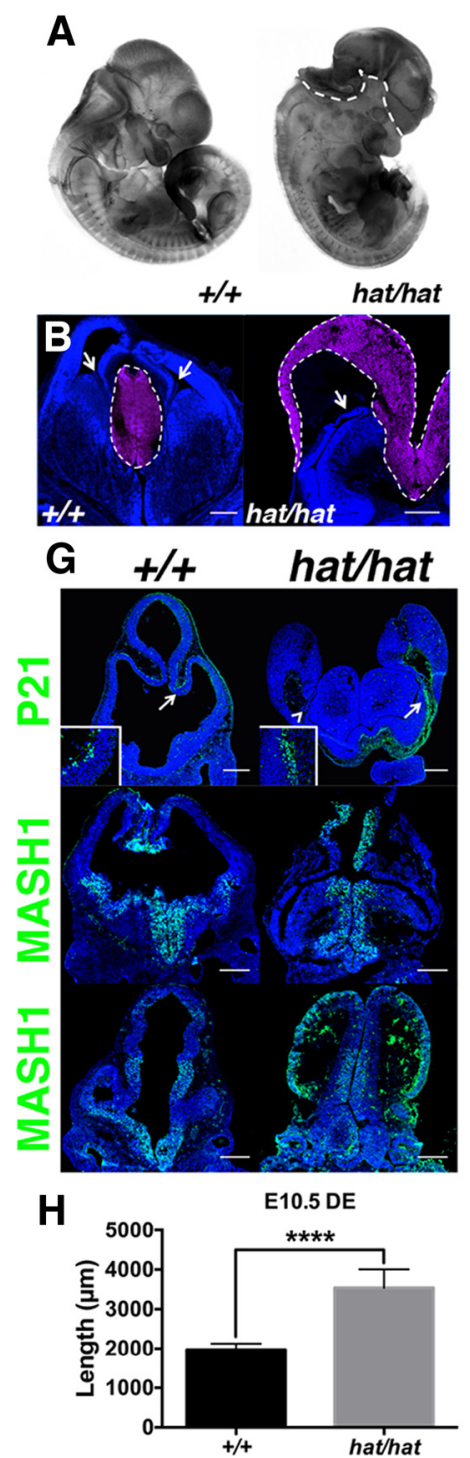

C

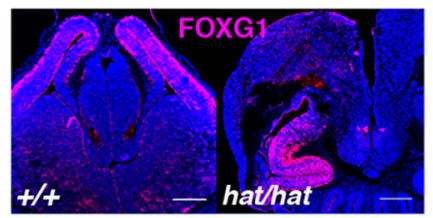

D
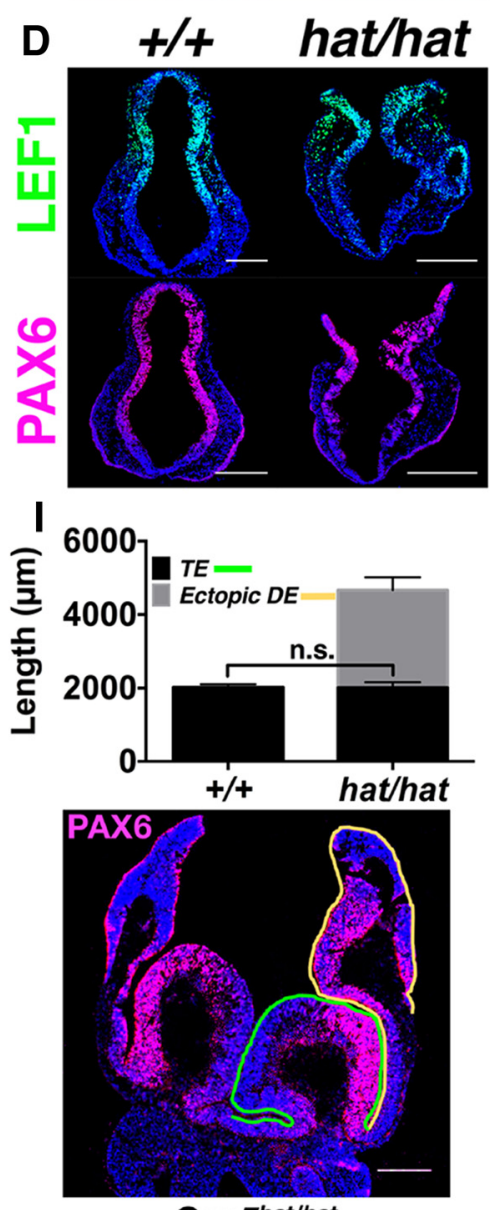

Gcn5 $5^{\text {hat } / h a t ~}$
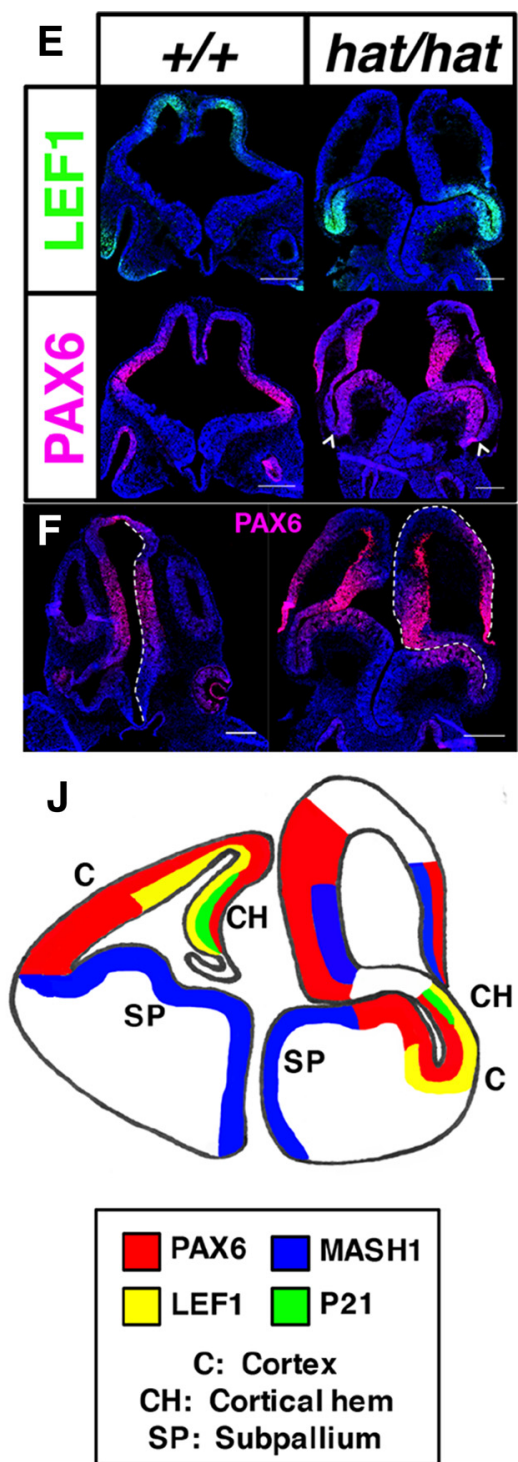

Figure 1. (Gcn $\left.5^{\text {hat } h a t}\right)$ embryos exhibit significant diencephalic expansion and telencephalic compression. A, Neurofilament-stained E11.5 wild-type and (Gcn $\left.5^{\text {hat } / \text { hat }}\right)$ embryos highlight the severe exencephaly exhibited by (Gcn $5^{\text {hat hat }}$ ) embryos. Dotted line indicates the dorsal margin of the everted neural tissue. $\boldsymbol{B}$, False-coloring based on morphology illustrates the normal relative sizes of the telencephalon (blue) and diencephalon (purple) at E12.5 in wild-type embryos $\left(+/+\right.$ ) and apparent expansion of the diencephalon in $\left(G c n 5^{\text {hat } h a t}\right.$ ) mutants. Arrows indicate the choroid plexus. Scale bars, $350 \mu \mathrm{m}$. $\boldsymbol{C}$, Immunostaining for FOXG1 atE12.5 indicates that the expanded tissue observed in mutants is not of telencephalic origin $(N=4)$. Scale bars, $350 \mu \mathrm{m} . \boldsymbol{D}$, E9.5 embryos indicate normal, dorsally restricted staining patterns of LEF1 (green) and PAX6 (magenta) in (Gcn $5^{\text {hat hat }}$ ) mutants $(N=4)$. Scale bars, $200 \mu \mathrm{m}$. E, Immunostaining for LEF1 and PAX6 at E10.5 in the region of the telencephalon (TE, rostral to the eyes in coronal sections) in wild-type embryos (left) shows restriction of telencephalic staining patterns to the dorsal midline and intermediate regions of the TE neuroepithelium, respectively $(N=4)$. Scale bars, 150 $\mu \mathrm{m}$. In (Gcn5 $\left.{ }^{\text {hat hat }}\right)$ mutants, LEF1 stops midway along the expanded tissue and PAX6 shows two domains of expression, with the ventral domain corresponding with the presumptive telencephalic limit (arrowheads), marked as well by LEF1.F, Staining for PAX6 in the diencephalon (DE; caudal eye region in coronal sections) in wild-type E10.5 embryos shows a similar pattern to that seen in the dorsally expanded tissue of the mutant TE region (analyzed along length of dotted line, $N=4)$. $G$, ( Gcn5 ${ }^{\text {hat } h a t}$ ) embryos at E11.5 show P21 expression in an intermediate region of the dorsal-ventral neuroepithelial axis in the TE region, which is correspondent with the cortical hem in wild-type embryos (arrows). Inset, Region indicated by arrows $(N=3)$. Mutantembryos also show a characteristic ribbon-liketissuethat is consistent with choroid plexus (arrowhead) located immediately dorsal to the same intermediate region. Scale bars, $150 \mu \mathrm{m}$. At E10.5, MASH1 staining in mutants is restricted to a ventral region of neuroepithelium that corresponds with the subpallium in wild-type embryos (middle panels). Scale bars, $150 \mu \mathrm{m}$. Dorsal MASH1 staining in mutants is consistent with staining observed in the wild-type diencephalon (bottom panels). Scale bars, $150 \mu \mathrm{m}$. $\boldsymbol{H}$, Measurements of dorsal-ventral length in the diencephalon (DE; caudal eye region in coronal sections) atE10.5 indicate significantly increased DE length in (Gcn ${ }^{\text {hat }}$ hat) ) mutants ( $N \geq 4$ embryos per genotype). I, Measurements of dorsal-ventral length of the telencephalic region in the rostral forebrain of (Gcn $5^{\text {hat } h a t}$ ) embryos indicate that the observed diencephalic expansion does not occur at the expense of telencephalic tissue. Telencephalic length in wild-type and mutant embryo sections was assessed via measurement of the neuroepithelium from the ventral most point of the neuroepithelium to the dorsal limit of PAX6-positive tissue (green line). Ectopic diencephalon was measured from the dorsal limit of the ventral domain of PAX6-positive tissue to the dorsal limit of the neuroepithelium (yellow line, $N \geq 5$ embryos per genotype). J, Schematic summarizing the staining patterns of PAX6, LEF1, MASH1, and P21 in the wild-type (left) and mutant (right) forebrain at E10.5. Scale bars, $200 \mu \mathrm{m}$. ${ }^{* * *} p<0.0001$. n.s., $p>0.05$.

GCN5 acetyltransferase activity ((Gcn5 $\left.\left.5^{\text {hat hat }}\right)\right)$ survive to approximately embryonic day 16.5 (E16.5) and display severe cranial neural tube defects (NTDs) (Bu et al., 2007) (Fig. 1A; E11.5, in all mutant embryo figures the dotted line indicates dorsal limit of the everted neural tissue). Gross morphological examination of $\left(G c n 5^{\text {hat } / h a t}\right)$ mutants indicated dramatic overgrowth of neural tissue compared with other cranial NTD models in our laboratory (Zohn et al., 2005; Zeng et al., 2010; Chen et al., 2012; Harmacek et al., 2014), suggesting a failure of negative regulatory mechanisms of neuroepithelial growth during early neural development. Surprisingly, analysis of sections through the developing forebrain at E12.5, when individual forebrain structures can be 
readily identified by morphology, revealed specific expansion of diencephalic structures in ( $G c n 5^{\text {hat } / \text { hat }}$ ) embryos (Fig. $1 B$, purple false-coloring) and subsequent compression, deformation, and ventral displacement of the cortex and choroid plexus (Fig. $1 B$, blue tissue and arrows). We further confirmed that this expansion is diencephalon-specific at this time point in $\left(G c n 5^{\text {hat } / h a t}\right)$ mutants via immunostaining for the telencephalic marker FOXG1, which did not stain any of the overgrown and everted neuroepithelium but did stain the deformed and displaced telencephalon (Fig. 1C).

To determine when these forebrain changes are first detected, we examined a set of forebrain patterning markers at E9.5 and E10.5. Despite the open neural tube, forebrain patterning appeared normal in E9.5 (Gcn5 $5^{\text {hat } / \text { hat }}$ ) embryos, as indicated by PAX6 and LEF1 immunohistochemical staining, which mark dorsal and intermediate regions, respectively (Fig. 1D). By E10.5, PAX6 and LEF1 mark the pallium and dorsal pallium/cortical hem, respectively (wild-type expression in left panels of Fig. 1E, schematic in Fig. 1J). PAX6 staining revealed an interesting change in patterning in $\left(G c n 5^{\text {hat } / \text { hat }}\right)$ embryos, with what appeared to be a normally graded, ventral-to-dorsal expression pattern that stopped at an intermediate domain along the dorsalventral axis (Fig. 1E, staining ventral to arrowheads) and two new domains in the dorsal half of the expanded neuroepithelium (Fig. $1 E$, dorsal to arrowheads). LEF1 staining revealed a similar pattern of positive expression in an intermediate domain along the dorsal-ventral axis but was absent from the dorsal half of the neuroepithelium. Because we observed expansion of diencephalic tissue at later stages of development and PAX6 is also expressed in the wild-type diencephalon, but LEF1 is not, we hypothesized that the tissue beyond the intermediate domain of PAX6 and LEF1 staining in Gcn5 mutants was of diencephalic origin. We therefore examined PAX6 staining in the wild-type diencephalon at E10.5 and found that the staining pattern in the expanded dorsal tissue in mutants was highly similar to that of the wild-type diencephalon (Fig. $1 F$, patterning along dotted lines). Similar results were found for P21, which is expressed specifically in the cortical hem at E11.5 and was only observed in an intermediate domain of $\left(G c n 5^{\text {hat } / h a t}\right)$ mutants at that time point (Fig. $1 G)$. Staining for MASH1, which is expressed in a specific pattern in the diencephalon, revealed a similar, albeit more variable, pattern to that of PAX6 in the dorsally expanded tissue (Fig. 1G). These staining results are summarized in Figure $1 J$ with wild-type schematized on the left side and Gcn 5 mutant on the right side.

The timing of the onset of the diencephalic overgrowth was examined in sectioned E9.5 and E10.5 (Gcn5 $\left.5^{\text {hat } / \text { hat }}\right)$ embryos compared with wild-type by analyzing the dorsal-ventral length of the neuroepithelium in the telencephalic (TE) and diencephalic (DE) regions. In accordance with the molecular marker analyses above, the length measurements $(\mu \mathrm{m})$ revealed normal TE and DE size in mutant embryos at E9.5 (Gcn5 $5^{+/+}$TE, $970 \pm$ $61, N=4 ;\left(G c n 5^{\text {hat } / \text { hat }}\right)$ TE, $940 \pm 68, N=4$; unpaired $t$ test, $p=$ 0.832; Gcn $5^{+/+}$DE, $990 \pm 20 ;\left(G c n 5^{\text {hat } / \text { hat }}\right)$ DE, $984 \pm 34 ; p=$ 0.967 ), but significant overgrowth of the DE at E10.5 (Fig. $1 H$; Gcn $5^{+/+}, 1972 \pm 155 N=6$; (Gcn5 $\left.5^{\text {hat } / h a t}\right), 3538 \pm 467, N=5$; unpaired $t$ test, $\left.p=1.7 \times 10^{-13}\right)$. To address whether the diencephalic expansion in $\left(G c n 5^{\text {hat } / \text { hat }}\right)$ mutants comes at the expense of telencephalic tissue, we characterized tissue in the TE region as either telencephalon or ectopic diencephalon based on its relationship to the dorsal limit of LEF1 staining (Fig. 1I, TE in green, ectopic DE in yellow in the tissue section). Measurement of the TE from its ventral limit to the dorsal limit of LEF1 staining indicated that Gcn5 mutants have a TE of normal length, al- though it is severely compressed $\left(G c n 5^{+/+}, 2025 \pm 82, N=6\right.$; $\left(G c n 5^{\text {hat } / \text { hat }}\right), 2015 \pm 150, N=5$, unpaired $t$ test, $\left.p=0.890\right)$. Therefore, the significant expansion of the neuroepithelium observed in the TE region of (Gcn $\left.5^{\text {hat } / h a t}\right)$ embryos is due solely to the existence of ectopic DE located dorsal to the TE. Together, these data show that (Gcn $5^{\text {hat } / \text { hat }}$ ) embryos exhibit specific expansion of the diencephalon (Fig. 1I, gray bar) and that the rostral expansion of the diencephalon beyond its normal borders leads to compression of the telencephalic tissue. These data together with our patterning experiments indicate that the expansion of the telencephalic region is a consequence of diencephalic expansion beyond its normal domain, rather than a true expansion of the telencephalon. The summary of the mutant phenotype shown in Figure $1 J$ therefore suggests that the forebrain tissue expansion in $\left(G c n 5^{\text {hat } / \text { hat }}\right)$ mutants is caused by the failure of a negative regulatory mechanism controlling diencephalic size, indicating a temporal- and tissue-specific function for GCN5 in the developing diencephalon that begins between E9.5 and E10.5.

\section{Dysregulation of diencephalic signaling pathways in (Gcn5 $\left.5^{\text {hat/hat }}\right)$ mutants}

During normal forebrain development, the onset of diencephalic growth and patterning is accompanied by specific upregulation of Shh expression in a region known as the zona limitans intrathalamica (ZLI; the finger-like projection is marked by an asterisk in Fig. 2A, left) (Scholpp and Lumsden, 2010). As development progresses, the ZLI-associated $\mathrm{SHH}$ signaling acts as both a mitogen and a morphogen to regulate the size of the diencephalon and pattern the developing thalamic nuclei, respectively (Vue et al., 2009; Haddad-Tóvolli et al., 2012). Because of the important mitogenic role of $\mathrm{SHH}$ in the diencephalon and the increased diencephalic size in $\left(G c n 5^{\text {hat } / h a t}\right)$ mutants highlighted in Figure 1, we hypothesized that Shh expression is increased in the diencephalon of $\left(G c n 5^{\text {hat } / h a t}\right)$ embryos. Indeed, $\left(G c n 5^{\text {hat } / h a t}\right)$ embryos exhibit a significant expansion of Shh expression along both the rostral-caudal and dorsal-ventral axes that correlates closely with the expanded diencephalic tissue (Fig. 2A, right, rostral-caudal expansion denoted by red dotted line). Studies in chick have demonstrated that repression of Gli3 in the alar plate is required to render the presumptive ZLI region permissive to dorsal Shh expansion (Martinez-Ferre et al., 2013); and in accordance, we observed decreased Gli3 expression in (Gcn5 $\left.5^{\text {hat } / \text { at }}\right)$ mutants (Fig. 2B). qRT-PCR for Shh and Gli3 on forebrain tissue from wild-type and mutant embryos at E10.5 also showed significant, albeit modest, upregulation of Shh and downregulation of Gli3 transcript levels (Fig. 2E; Gcn $5^{+/+}$Shh, $1.00 \pm 0.107$; $\left(G c n 5^{\text {hat } / \text { hat }}\right), 1.334 \pm 0.0782 ; N=4$, unpaired $t$ test, $p=0.0240$; Gcn $5^{+/+}$Gli3, $1.00 \pm 0.0489 ;\left(G c n 5^{\text {hat } / \text { hat }}\right), 0.564 \pm 0.191 ; N=4$, unpaired $t$ test, $p=0.005)$. The modest changes in expression observed by qRT-PCR are likely due to the complex mixture of cells in the isolated tissue, the small size of the domain of Gli3 expression surrounding the ZLI, and the significant expansion of the mutant tissue. Together, with the spatial resolution provided by in situ hybridization, the data in Figures 1 and 2 strongly support the requirement of GCN5 activity in restriction of diencephalic SHH signaling.

WNT signaling promotes diencephalic Shh expression through repression of Gli3 (Martinez-Ferre et al., 2013), but the WNT ligands important for diencephalic patterning in mouse are still unclear. To investigate whether $\left(G c n 5^{\text {hat } / \text { hat }}\right)$ mutants exhibit increased WNT signaling in the forebrain at the time of ZLI expansion, we performed in situ hybridization for Axin2, which provides a reliable readout of WNT signaling (Jho et al., 2002). Although Axin2 levels are low in 
the diencephalon in wild-type embryos, levels of Axin 2 transcript are robustly increased in the expanded diencephalic tissue of mutants (Fig. 2C), a finding that was confirmed by qRT-PCR (Fig. 2E; Gcn $5^{+/+}, 1.00 \pm$ 0.0649; (Gcn5 $\left.5^{\text {hat } / \text { hat }}\right), 1.935 \pm 0.687 ; N=4$, unpaired $t$ test, $p=0.035)$. We also examined the expression levels of Wnt8b, which has been shown to repress diencephalic Gli3 expression in chick. Again, both in situ hybridization and qRT-PCR revealed significant upregulation of Wnt8b in mutants (Fig. 2D, E; Gcn $5^{+/+}, 1.00 \pm 0.0449$; $\left(G c n 5^{\text {hat/hat }}\right), 1.864 \pm 0.483 ; N=4$, unpaired $t$ test, $p=0.037$ ), suggesting broad activation of the WNT signaling pathway in the forebrains of ( Gcn $\left.5^{\text {hat } / \text { hat }}\right)$ embryos. Together, these data support a mechanism by which GCN5 restricts diencephalic expansion through repression of WNT signaling, which acts upon Gli3 to limit Shh expression. In support of this model, qRT-PCR analysis of Shh, Gli3, $W n t 8 b$, and Axin 2 expression in forebrain tissue at E9.5, a time when mutant embryos display normal forebrain size (Fig. $1 D)$, revealed significant upregulation of Wnt8b and Axin2 in mutants, but no significant changes in Shh and Gli3 (Fig. 2F; Gcn $5^{+/+}$Axin2, $1.00 \pm 0.100$; $\left(\right.$ Gcn $\left.5^{\text {hat } / \text { hat }}\right), 1.631 \pm 0.278 ; N=3$, unpaired $t$ test, $p=0.046$; Gcn $5^{+/+}$Wnt8b, $1.00 \pm 0.060 ;\left(G c n 5^{\text {hat } / \text { hat }}\right), 1.832 \pm 0.250 ; N=3$, unpaired $t$ test, $p=0.024)$. This suggests that dysregulation of WNT signaling precedes dysregulation of SHH signaling, which supports the hierarchy of signaling pathways defined by others, as well as our hypothesis that upregulation of $\mathrm{SHH}$ signaling is the mitogenic factor driving diencephalic expansion in Gcn5 mutants.

Our observation that loss of GCN5 enzymatic activity leads to disrupted expression of key regulators of diencephalic size and patterning led us to revisit the patterning of diencephalic domains in $\left(G c n 5^{\text {hat } / h a t}\right)$ mutants to determine whether there are region-specific changes. NKX2.2, OLIG2, PAX6, and PAX7 are patterning molecules that are restricted to specific diencephalic domains during development. Immunostaining at E10.5 for NKX2.2, which marks the prethalamus and rostral thalamus, and OLIG2, which marks the prethalamus and rostral region of the caudal thalamus, revealed significant expansion of both domains, as well as mispatterning highlighted by nonoverlapping expression in the dorsal diencephalon and a lack of the dorsal-most NKX2.2-positive domain (Fig. 3A, top panels). To better understand the extent of this phenotype, we examined costaining for PAX6 and OLIG2 in the telencephalic region of $\left(G c n 5^{\text {hat } / h a t}\right) \mathrm{mu}-$ tants at E10.5 and found that the ectopic DE region identified in Figure 1 is positive for OLIG2 (Fig. $3 A$, middle panels), suggesting a significant dorsal and rostral expansion of the prethalamus that is in line with the rostrally expanded Shh expression observed in Figure $2 A$ (dotted red line). (Gcn $\left.5^{\text {hat } / \text { hat }}\right)$ embryos also lack PAX7 staining in the dorsal diencephalon at E10.5, indicating a delay or misspecification of the developing pretectum and/or epithalamus (Fig. 3A, bottom panels). Examination of NKX2.2 and OLIG2 staining in mutants at E12.5 revealed similar patterns of dysregulation and an increase in the distance between the prethalamus and rostral domain of the caudal thalamus, as indicated by an increased distance between OLIG2-positive domains and greater spacing between the NKX2.2 domains, as well as a significant weakening of the dorsal-most NKX2.2 domain (Fig. $3 B$, top and middle panels). Surprisingly, (Gcn5 $\left.5^{\text {hat } / \text { hat }}\right)$ embryos show positive PAX7 staining at E12.5 along with an increase in the size of the PAX7 domain compared with wild-type embryos (Fig. 3B, bottom panels), suggesting that PAX7 induction in the pretectum is delayed in mutants, but that the pretectum expands at a significantly increased rate once it is specified. Together, these findings support a role for GCN5 in the specification, patterning, and spacing of the dorsal and rostral diencephalon.

We next asked whether the expansion and disrupted patterning of the rostral thalamus, prethalamus, and pretectum correspond with the expanded ZLI observed in Figure 2. Because of the degree of tissue folding in mutants, it is difficult to assess the extent of Shh expansion along the dorsal-ventral axis in whole-mount in situ hybridization. We therefore performed in situ hybridization on sections from wild-type and mutant embryos at E10.5. Probing for Shh transcript revealed that mutant embryos have a significant expansion of the ZLI along the dorsal-ventral axis in the region corresponding to the mispatterned thalamic nuclei represented by OLIG2 and NKX2.2 (Fig. 3C). In other words, the increased spacing observed between the prethalamus and rostral portion of the caudal thalamus in $\left(G c n 5^{\text {hat } / \text { at }}\right)$ embryos is due to an expansion of the ZLI, which lies between these two domains. We were surprised to observe a loss of Shh in the basal plate, as our staining experiments revealed normal patterning of the ventral diencephalon. However, previous work has shown that Shh induction and regulation in the ZLI can occur independently of Shh in the ventral midline (Guinazu et al., 2007). This is an aspect for future study, but with respect to the focus 
A

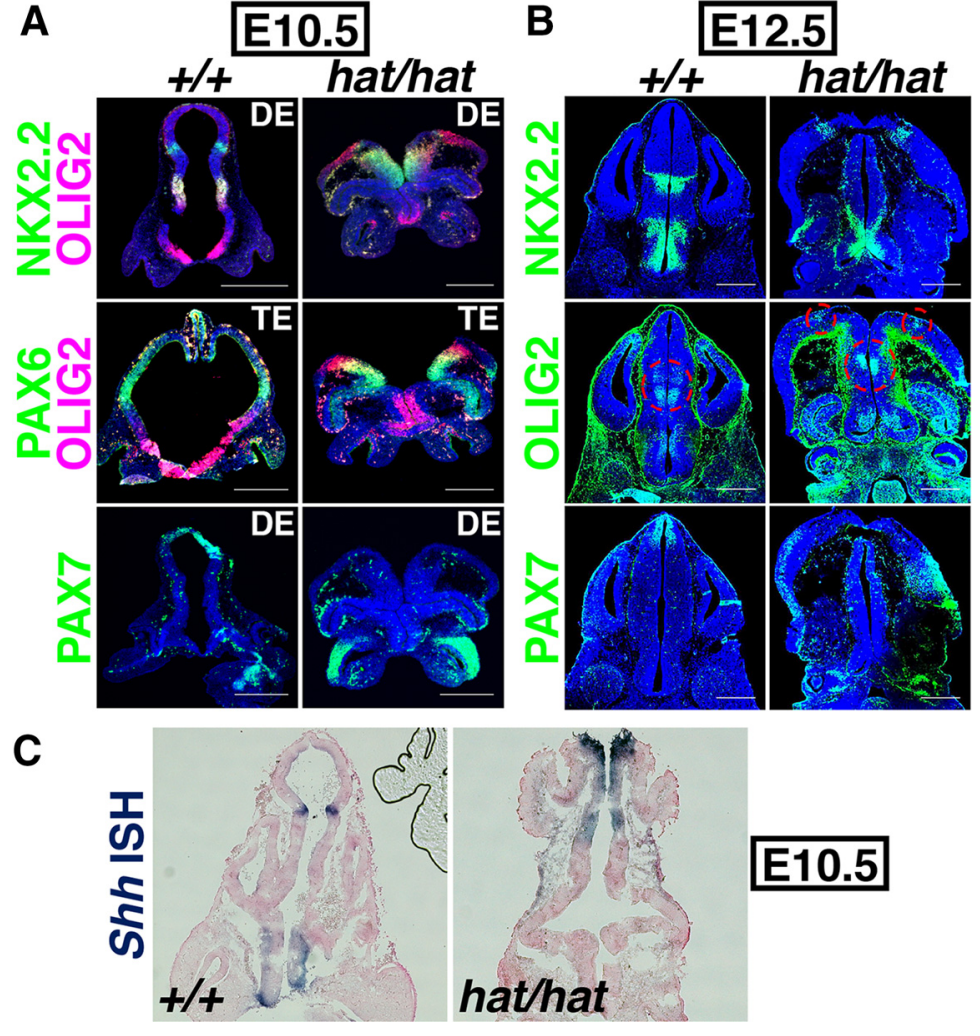

Figure 3. Mispatterning and expansion of the developing diencephalon in $\left(G \mathrm{cn} 5^{\text {hat } / \text { hat }}\right)$ mutants. $A$, Immunohistochemistry for NKX2.2 (green) and 0LIG2 (magenta) at E10.5 indicates significant expansion and mispatterning of both markers in the rostral diencephalon (top panels). Staining for PAX6 (green) and OLIG2 (magenta) in the telencephalic region shows significant rostral expansion of the prethalamus, as indicated by the presence of a large, dorsal 0LIG2-positive domain in mutant, but not wild-type embryos (middle panels). PAX7 (green) staining is absent in the dorsal diencephalon of ( $\mathrm{GCn} 5^{\text {hat } / \text { hat }}$ ) mutants, indicating mispatterning or a delay in dorsal tissue specification (bottom panels, $N=3$ animals per condition). Scale bars, $250 \mu \mathrm{m}$. $\boldsymbol{B}$, Immunostaining for NKX2.2 (top panels) shows increased spacing along the dorsal-ventral axis between NKX2.2-positive domains in mutants and decreased staining in the dorsal-most domain. Staining for 0LIG2 (middle panels) shows an increase in the distance between the prethalamus and rostral region of the caudal thalamus in mutants (dotted red circles). Immunohistochemistry for PAX7 (bottom panels) at E12.5 shows a significant increase in the size of the pretectum in $\left(G c n 5^{\text {hat }}{ }^{\text {hat }}\right)$ mutants $(N=3)$. Scale bars, $500 \mu \mathrm{m}$. C, Section in situ hybridization for Shh at E10.5 shows significant expansion of the ZLI, indicated by positive Shh signal that also appears to be increased in intensity, along the dorsal-ventral axis $(N=3)$.

of our current studies, experiments have shown that only signals from the ZLI, and not the basal plate, are necessary for diencephalic patterning (Vieira and Martinez, 2006). Together, our data reveal a significant expansion of the ZLI along both the dorsal-ventral (Fig. $3 C$ ) and rostral-caudal (Fig. $2 A$ ) axes that drives overgrowth of the dorsal diencephalon and expansion and mispatterning of the rostral thalamus, prethalamus, and pretectum.

\section{GCN5 activity is required for diencephalic RA signaling}

The significant upregulation of WNT signaling in (Gcn $\left.5^{\text {hat hat }}\right)$ forebrains led us to investigate possible upstream regulators of Wht and Shh in the diencephalon. Previous studies in chick identified the noncanonical RA synthesizing enzyme CYP1B1 as a potential repressor of SHH in the alar plate (Chambers et al., 2007). Expression of Cyp $1 b 1$ turns on at the time of ZLI-associated SHH expansion and ectopic expression of GFP-tagged CYP1B1 is sufficient to repress Shh in the diencephalon, even through non-cell-autonomous mechanisms (Chambers et al., 2007). Additionally, RA has been shown to repress WNT signaling in multiple contexts (Easwaran et al., 1999; Shah et al., 2003; S. Li et al., 2008). GCN5 has been implicated in positive regulation of RA signaling in vitro through its interaction with RAR $\alpha$ via the SAGA complex member ADA3, which directly binds to the RAR $\alpha$ coactivator receptor pocket (Brown et al.,
2003; C. W. Li et al., 2010). Forebrainderived RA has a role in cortical migration (Choi et al., 2014), generation of cortical neurons (Siegenthaler et al., 2009; Harrison-Uy et al., 2013), and development of the choroid plexus (Gupta and Sen, 2015); however, it has been concluded that RA signaling is dispensable for gross structural development of the mouse forebrain before E12.5 (Molotkova et al., 2007). Nonetheless, compound mutant mice lacking both $\operatorname{RAR} \alpha$ and $\operatorname{RAR} \gamma$ display a significant lengthening of the neuroepithelium and compression of the telencephalon (Lohnes et al., 1994), a phenotype highly similar to that seen in (Gcn5 $\left.5^{\text {hat } / \text { at }}\right)$ embryos. Lohnes et al. (1994) argued that this phenotype due to loss of RAR $\alpha$ and RAR $\gamma$ is caused by impaired accumulation of CSF and ventricular collapse due to hindbrain exencephaly. However, our extensive experience with exencephaly mutants indicates that hindbrain exencephaly does not cause ventricular collapse in the forebrain and does not result in the characteristic neuroepithelial expansion and compression of the telencephalon observed in both $\left(G c n 5^{\text {hathat }}\right)$ and $\operatorname{Rar\alpha }^{-/-}$; Rar $\gamma^{-1-}$ embryos. Additionally, although mice lacking Raldh $3^{-/-}$, a well-characterized RA-synthesizing enzyme expressed in the forebrain, show normal cortical morphology at E10.5, Molotkova et al. (2007) did not discuss the diencephalic expansion observed in these embryos. Thus, a direct role for RA in development of the diencephalon remains controversial and has not been well studied in mammals. Furthermore, a potential role of GCN5 in RA-mediated forebrain development has not been studied. These previously published observations together with our data led us to hypothesize that GCN5 restricts WNT signaling and Shh expression in the developing diencephalon through direct promotion of RA signaling.

To test our hypothesis, we first crossed Gcn $5^{\text {hat/ }}$ mice with an RA reporter line harboring multiple RAREs upstream of the lac $Z$ gene (RAREhsplacZ) (Rossant et al., 1991). Wild-type mice with the RA signaling reporter display robust $\beta$-galactosidase staining in the telencephalon, as previously reported (Rossant et al., 1991), as well as in two distinct regions of the dorsal diencephalon that flank the ZLI (Fig. 4A, left, $B$, RA reporter diencephalic domains are marked by arrows, Shh expression in the ZLI is marked by an asterisk). Additionally, the retinoic acid synthesizing enzyme Raldh 3 is expressed in a highly similar pattern to that of the RAREhsplacZ reporter in the dorsal diencephalon (Molotkova et al., 2007). (Gcn5 $\left.{ }^{\text {hat } / \text { at }}\right)$;RAREhsplacZ embryos show a significant reduction in RA reporter activity in the diencephalon (Fig. $4 A$, right), supporting the hypothesis that GCN5 is required for diencephalic RA signaling. Experiments in chick have shown that the p3 and p2 domains of the developing diencephalon, which flank the ZLI and correspond with the diencephalic domains of RA signaling that are lost in $G c n 5$ mutants, are required to restrict Shh expression, as removal of either is sufficient to broadly expand Shh expression in diencephalic explants (Guinazu et al., 


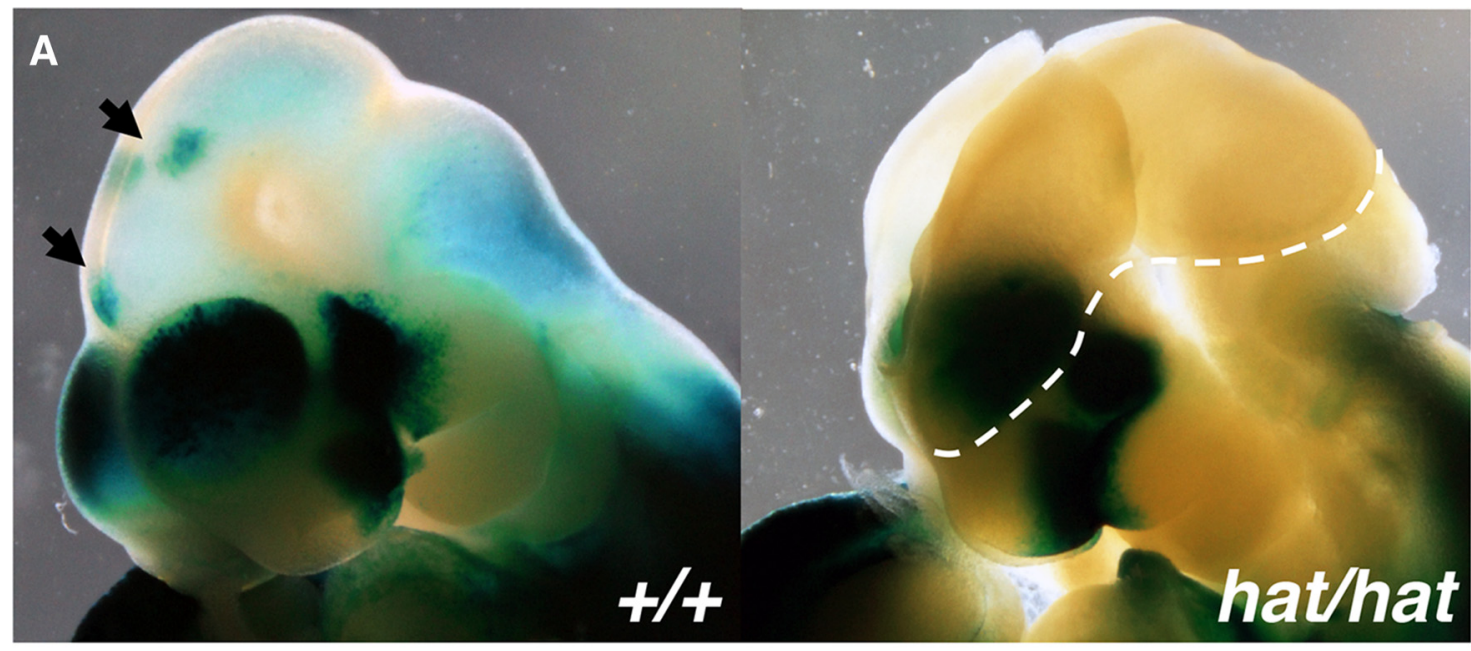

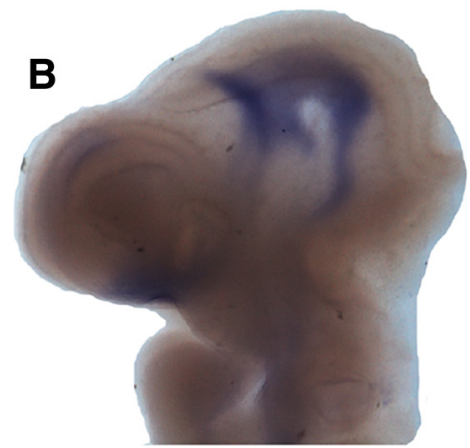

Shh ISH (Embryo 1)

C

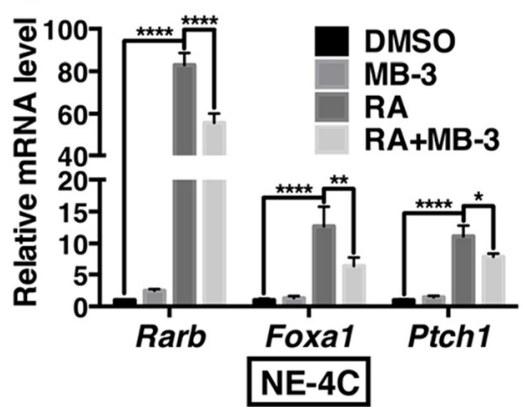

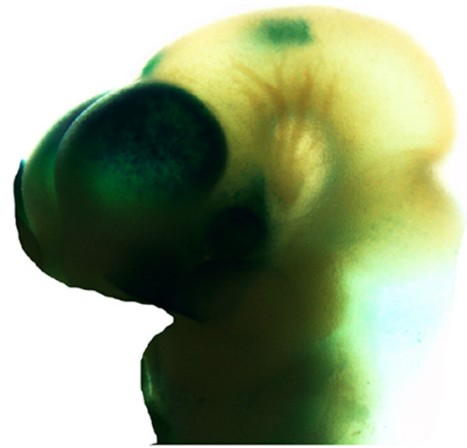

RAREhsplacZ (Embryo 2)

D

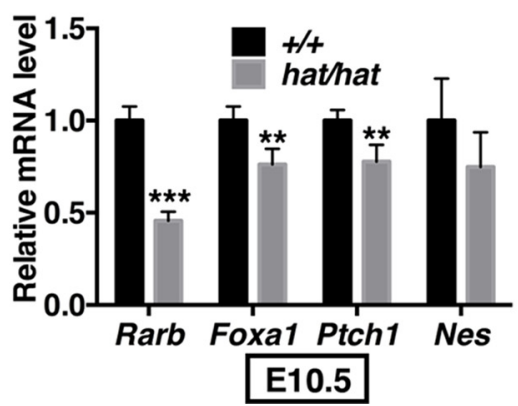

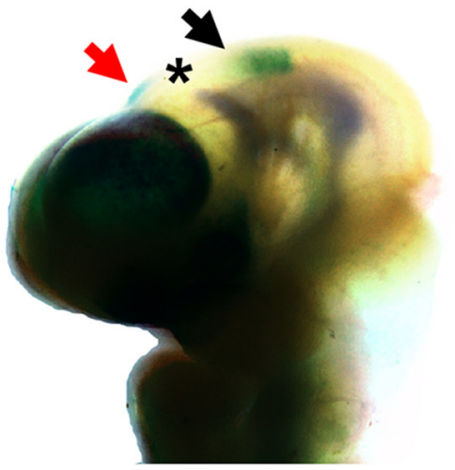

Merge

E

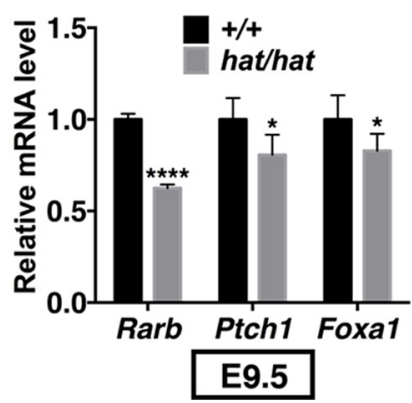

Figure 4. GCN5 acetyltransferase activity is required for RA signaling in the developing forebrain. $A$, Wild-type E10.5 RAREhsplacZ embryos show strong $\beta$-gal staining in the telencephalon, eye, and facial prominence, as well as in two distinct regions of the dorsal diencephalon that flank the alar plate (arrows). E10.5 (Gcn5 hat/hat);RAREhsplacZ embryos maintain RA signaling in the telencephalon, facial prominence, and eye but lack RA signaling in the dorsal diencephalon. Dotted line indicates dorsal limit of open neural tissue $(N=6$ embryos). $\boldsymbol{B}, 0 \mathrm{verlay}$ of an E10.5 $\mathrm{Gcn} 5^{+/+} ;$RAREhsplacZ embryo stained for lacZ onto a somite-matched Gcn $5^{+/+}$embryo that has undergone whole-mount in situ hybridization for Shh indicates that the regions of active RA signaling in the diencephalon (arrows) flank the ZLI along the rostral-caudal axis (asterisk). Furthermore, the rostral site of active RA signaling (red arrow) is positioned in line with the orientation of the extending ZLI. C, qRT-PCR using RNA isolated from NE-4C cells treated with $0.1 \mu \mathrm{m}$ RA for 16 h shows robust expression of Rarb, Foxa1, and Hoxb1, all of which are significantly downregulated by inhibition of GCN5 enzymatic activity with $100 \mu \mathrm{m} \mathrm{MB-3} \mathrm{(} N=4$ wells per condition). D, qRT-PCR using RNA isolated from wild-type and ( $G$ cn 5 hat/hat) forebrain tissue at E10.5 indicates significant downregulation of multiple RA target genes ( $N=4$ embryos per genotype). $\boldsymbol{E}$, qRT-PCR using RNA isolated from wild-type and (Gcn5 ${ }^{\text {hat/hat }}$ ) forebrain tissue at E9.5 indicates significant downregulation of Rarb, Ptch1, and Foxa1 ( $N=4$ embryos). ${ }^{*} p<0.05 .{ }^{* *} p<0.01 .{ }^{* * *} p<0.001 .{ }^{* * * *} p<0.0001$.

2007). The expansion of Shh expression coupled with the loss of RA signaling in Gcn 5 mutants further supports a role for RA signaling in restriction of diencephalic expansion.

To test the molecular relationship between GCN5 enzymatic activity and RA signaling, we first turned to the RA-responsive $\mathrm{NE}-4 \mathrm{C}$ neuroepithelial cell line that was originally isolated from E9.0 mouse forebrain (Schlett and Madarász, 1997). We coupled this with MB-3, a pharmacological inhibitor with specificity for GCN5 (GCN5 IC $_{50}=100 \mu \mathrm{M}$, CBP IC $50=500 \mu \mathrm{M}$ ) (Biel et al., 2004). Analysis of two bona fide RA target genes Rarb and Foxal (Jacob et al., 1999; Merrill et al., 2004; Lalevée et al., 2011) that are expressed in the forebrain and can be used as proxies of active RA signaling (Yamagata et al., 1994; Besnard et al., 2004; Mavromatakis et al., 2011) showed a significant reduction in RA- 
mediated transcription upon treatment with $100 \mu \mathrm{M}$ MB-3 (Fig. $4 C$; Rarb expression in RA vs RA + MB-3 95\% CI of difference = 23.46 to 31.06, two-way ANOVA, Tukey's multiple-comparisons test, adjusted $p<0.0001$; Foxal RA vs RA + MB-3 95\% CI of difference $=2.573$ to 10.180 , adjusted $p=0.0003$ ). We also observed a statistically significant decrease in expression of the Shh repressor Ptch1 (Fig. 4C; RA vs RA + MB-3 95\% CI of difference $=0.5302$ to 5.910 , adjusted $p=0.013$ ) Although Ptch1 is a target of SHH signaling, the Ptch1 locus contains an RARE that is regulated by RA and Ptch1 induction can occur independently of $\mathrm{HH}$ or Smoothened (Busch et al., 2014). These changes in RA target gene expression were also reflected in vivo, as forebrain tissue of E10.5 (Gcn5 $\left.5^{\text {hat } / \text { hat }}\right)$ mutants subjected to qRT-PCR revealed decreased expression of Rarb, Foxa1, and Ptch1 (Fig. 4D; $N=4$ for both genotypes, unpaired $t$ test, Rarb Gcn $5^{+/+}, 1.00 \pm$ 0.0754; (Gcn5 $\left.5^{\text {hat } / \text { hat }}\right), 0.4568 \pm 0.0492, p=1.97 \times 10^{-5} ;$ Foxal $G c n 5^{+/+}, 1.00 \pm 0.0751 ;\left(G c n 5^{\text {hat } / \text { hat }}\right), 0.7645 \pm 0.0836, p=$ 0.006; Ptch1 Gcn5 $5^{+/+}, 1.00 \pm 0.0574 ;\left(G c n 5^{\text {hat } / \text { hat }}\right), 0.7799 \pm$ $0.0895, p=0.006$ ). However, mutants did not show a significant decrease in Nes expression (Fig. 4D; Gcn $5^{+/+}, 1.00 \pm 0.225$; $\left.\left(G c n 5^{\text {hat } / \text { hat }}\right), 0.7504 \pm 0.1868, p=0.139\right)$, which also contains a putative RARE in its promoter (Balmer and Blomhoff, 2002; Lalevée et al., 2011), suggesting that a subset of RA-responsive genes and/or RAREs are not under GCN5-mediated control. This point is further supported by the persistence of lacZ staining in the telencephalic regions of ( $G c n 5^{\text {hat/hat }}$ ) mutants (Fig. $\left.4 A\right)$. Because we observed increased WNT signaling in mutants at E9.5, we then asked whether $\left(G c n 5^{\text {hat } / \text { hat }}\right)$ embryos exhibit disrupted RA signaling at the same developmental time point. Indeed, there was significant downregulation of the RA target genes Rarb, Ptch1, and Foxal (Fig. 4E; $N=4$ for both genotypes, unpaired $t$ test, Rarb Gcn5 $5^{+/+}, 1.00 \pm 0.0304 ;\left(G c n 5^{\text {hat/hat }}\right), 0.6247 \pm 0.0202$, $p=2.55 \times 10^{-8} ;$ Ptch 1 Gcn5 $5^{+/+}, 1.00 \pm 0.106 ;\left(G c n 5^{\text {hat } / \text { hat }}\right)$, $0.8063 \pm 0.110, p=0.044 ;$ Foxal Gcn $5^{+/+}, 1.00 \pm 0.111$; $\left.\left(G c n 5^{\text {hat } / \text { hat }}\right), 0.8286 \pm 0.0724, p=0.042\right)$. Together, these data demonstrate that GCN5 enzymatic activity is required for diencephalic RA signaling and robust transcription of RA target genes. Furthermore, the diencephalic expansion and telencephalic compression observed in (Gcn $\left.5^{\text {hat/hat }}\right)$ embryos is strikingly similar to that seen in $\mathrm{Rara}^{-/-} ; \mathrm{Rarg}^{-/-}$embryos (Lohnes et al., 1994), suggesting that RA-mediated signaling acts in conjunction with GCN5 enzymatic activity to restrict the size of the diencephalon and maintain its morphology. Last, loss of GCN5 enzymatic activity results in decreased forebrain RA signaling by E9.5, which coincides with increased WNT signaling and precedes noticeable changes in $\mathrm{SHH}$ signaling and diencephalic size.

\section{GCN5-mediated acetylation of TACC1 regulates an RAR $\alpha$ / GCN5/TACC1 complex}

We next addressed the mechanism by which GCN5 regulates RA signaling in the forebrain. While GCN5 is best characterized as a histone acetyltransferase, numerous studies have suggested that many tissue-specific functions of GCN5 are performed through acetylation of nonhistone proteins (Jacob et al., 2001; Lerin et al., 2006; Conacci-Sorrell et al., 2010; Carradori et al., 2011; Zelin et al., 2012; L. Li et al., 2015). Mining of the literature for potential nonhistone targets of GCN5 that are expressed in the forebrain and involved in RA signaling identified the poorly characterized protein TACC1 (Gabillard et al., 2011). GCN5 and TACC1 can physically interact (Gangisetty et al., 2004), but the functional basis of this interaction is unclear. TACC1 is strongly expressed in the developing forebrain neuroepithelium at E10.5 (Lauffart et al., 2006) and has also been shown to physically interact with $\operatorname{RAR} \alpha$ and play a role in
RAR $\alpha$-mediated signaling (Guyot et al., 2010). We therefore hypothesized that GCN5 regulates RA signaling in the diencephalon through a mechanism involving direct acetylation of TACC1. Using NE-4C cells treated with RA, we assayed TACC1 acetylation in cytoplasmic and nuclear fractions via immunoprecipitation using an antiacetylated lysine antibody and subsequent Western blotting for TACC1. Acetylated TACC1 levels were then normalized to total TACC1 levels. Control and MB-3-treated NE-4C cells showed almost undetectable levels of acetylated TACC1 in both cellular fractions, but treatment of the cells with RA induced robust TACC1 acetylation, specifically in the nuclear fraction (Fig. $5 A$; nuclear and cytoplasmic controls represented by POLII and $\beta$-TUB, respectively). Consistent with our hypothesis, concurrent treatment of NE-4C cells with RA and MB-3 inhibited acetylation of TACC1, suggesting that GCN5 acetyltransferase activity is required for RAmediated acetylation of TACC1 (Fig. 5A). These results were further confirmed using protein extracted from forebrain tissue of E10.5 embryos, which consistently showed decreased levels of acetylated TACC1 in $\left(G c n 5^{h a t / h a t}\right)$ mutants compared with wild-type (Fig. 5B; $\left(G c n 5^{\text {hat } / \text { at }}\right)$ ac-TACC1 normalized to total TACC1 and relative to wild-type is $0.454 \pm 0.067, N=3$ embryos per genotype, unpaired $t$ test, $p=0.015$ ).

It has been suggested that activation of RA target genes by RAR $\alpha$ can occur in the absence of ligand under certain circumstances but that this ligand-independent signaling is negatively regulated by cell type-specific mechanisms (Park et al., 2010). This made us question whether a tissue-specific regulatory mechanism exists in the developing forebrain, perhaps reflected by poised RAR $\alpha$-GCN5-TACC1 complexes that localize to RA-responsive genes in the absence of RA as a means to increase transcriptional efficiency upon ligand binding. To test the existence of such a complex, we first performed immunoprecipitation of $\operatorname{RAR} \alpha$ on protein extracts from wild-type E10.5 forebrain tissue, which confirmed robust interactions between RAR $\alpha$, GCN5, and TACC1 (Fig. 5C). We then performed a series of coimmunoprecipitation experiments on nuclear fractions of NE-4C cells under varying conditions. Pull-down of RAR $\alpha$ revealed interaction with both GCN5 and TACC1 in the absence of RA that was unaffected by inhibition of GCN5 activity (Fig. 5D, lanes 8-10). Induction of neuronal differentiation with RA resulted in specific dissociation of TACC1 from the complex (Fig. 5D, lane 11). Interestingly, inhibition of GCN5 enzymatic activity with MB-3 during RA treatment stabilized this interaction (Fig. 5D, lanes 12-13), strongly suggesting that GCN5-mediated acetylation of TACC1 destabilizes the interaction of TACC1 with the RAR $\alpha$-GCN5 complex.

To investigate this complex further, we interrogated the genomic localization of $\operatorname{RAR} \alpha$, GCN5, and TACC1 in NE-4C cells before and after RA treatment via ChIP. Using primers flanking RAREs associated with Rarb and Foxal, we performed quantitative PCR of DNA immunoprecipitated with antibodies against RAR $\alpha$, GCN5, and TACC1. In the absence of RA, we found significant localization of all three proteins at both RAREs (Fig. 5E). Upon addition of RA, both RAR $\alpha$ and GCN5 were bound at the RAREs of both genes, but TACC1 was absent (Fig. 5E; Rarb RARE IgG vs TACC1 95\% CI of difference -3.513 to 1.295 , adjusted $p=0.605$; Foxa1 RARE IgG vs TACC1 95\% CI of difference -8.355 to 0.6226 , adjusted $p=0.112 ; N=4$ per condition, two-way ANOVA, Tukey's multiple-comparisons test). Inhibition of GCN5 activity with MB-3 significantly stabilized the interaction of TACC1 at both RAREs (Fig. 5E; Rarb RARE IgG vs TACC1 $95 \%$ CI of difference -11.63 to -6.826 , adjusted $p<$ 0.0001; Foxa1 RARE IgG vs TACC1 95\% CI of difference -30.52 to -21.54 , adjusted $p<0.0001 ; N=4$ per condition, two-way ANOVA, Tukey's multiple-comparisons test), suggesting that 

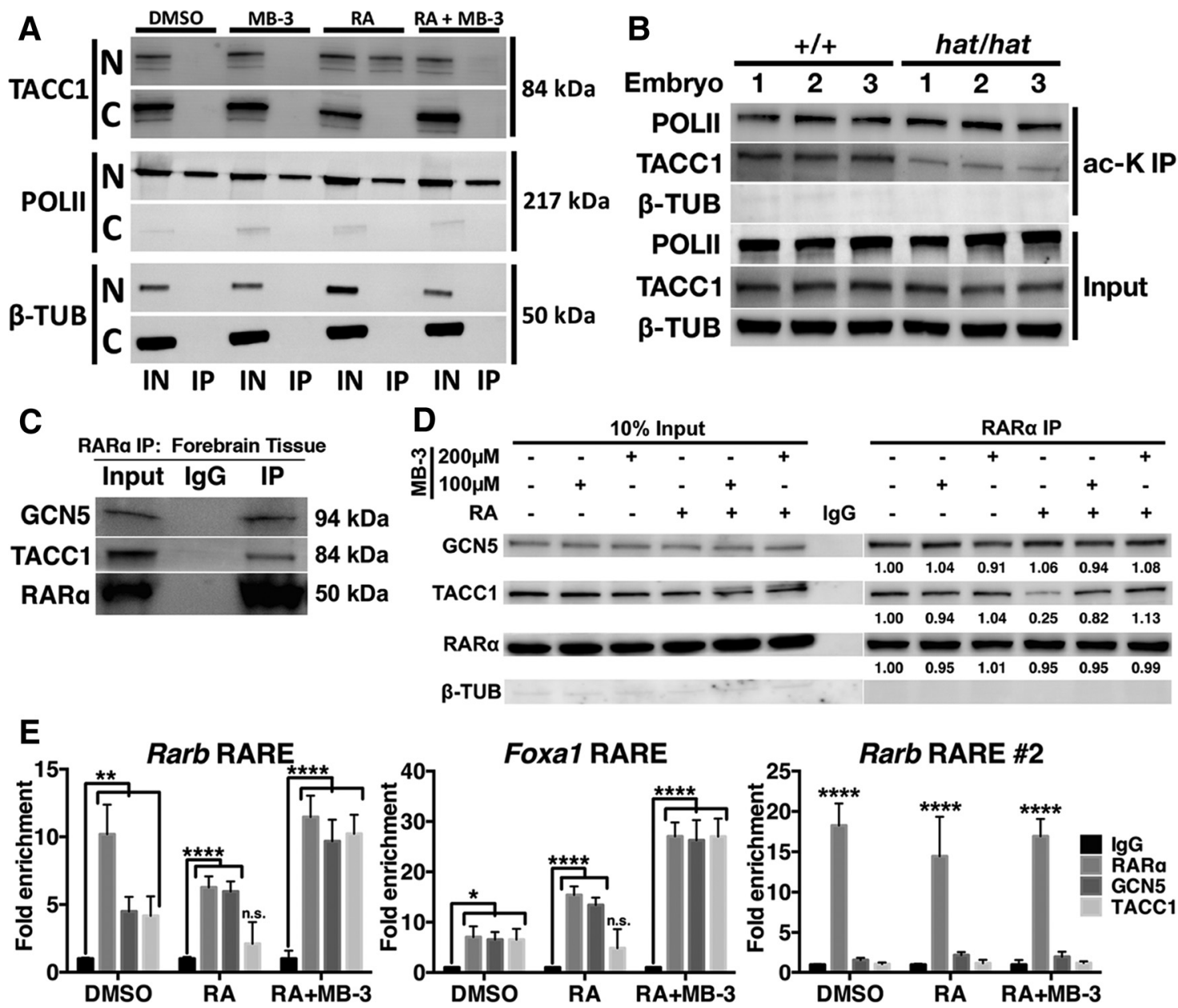

Figure 5. GCN5, RAR $\alpha$, and TACC 1 form an assembled RA-responsive complex on RAREs. A, Treatment of NE-4C cells with $0.1 \mu \mathrm{m}$ RA for $16 \mathrm{~h}$ induces robust acetylation of nuclear TACC 1 in a GCN5-dependent manner. POLII and $\beta$-TUB are shown as fractionation controls. POLII also serves as a positive control for immunoprecipitation, as a fraction of POLII is acetylated (N, Nuclear; C, cytoplasmic; $N=3)$. $\boldsymbol{B}$, Forebrain lysates of E10.5 (Gcn $\left.5^{\text {hat } / h a t}\right)$ embryos compared with wild-type embryos immunoprecipitated for acetylated lysine residues, followed by Western blotting for TACC1, POLII (positive IP control), and $\beta$-tubulin (negative IP control) indicates significantly decreased acetylation of TACC1 ( $N=3$ per genotype). C, Immunoprecipitation of RAR $\alpha$ from E10.5 forebrain extracts indicates interactions with both GCN5 and TACC 1 in vivo. D, Immunoprecipitation with anti-RAR $\alpha$ antibody shows association with GCN5 and TACC 1 in nuclear extracts of NE-4C cells. Treatment of NE-4C cells with $0.1 \mu \mathrm{m}$ RA disrupts association of TACC1, but not GCN5, with RAR $\alpha$. Inhibition of GCN5 catalytic activity stabilizes the TACC1-RAR $\alpha$ interaction in a dosedependent manner. Densitometry values indicated below the protein bands are normalized to input levels. $\beta$-Tubulin coimmunoprecipitation is displayed as a negative control $(N=3)$. $E$, ChIP demonstrates that RAR $\alpha, G C N 5$, and TACC1 are already associated with Rarb and Foxa1 RAREs before RA ligand treatment of NE-4C cells (control media with DMSO). Treatment with $0.1 \mu \mathrm{M}$ RA for $16 \mathrm{~h}$ causes specific dissociation of TACC 1 from the RAREs, but this dissociation is blocked by inhibition of GCN5 enzymatic activity with $100 \mu \mathrm{m}$ MB-3. ChIP for a second RARE associated with Rarb indicates a lack of GCN5 and TACC 1 binding $\left(N=4\right.$ wells per condition). ${ }^{*} p<0.05 .{ }^{* *} p<0.01$. ${ }^{* * *} p<0.0001$. n.s., $p>0.05$.

GCN5 acetyltransferase activity is required for the dissociation of TACC1 from target RAREs. In accordance with our previous conclusion that GCN5 only regulates a subset of RAREs, we did not observe binding of GCN5 and TACC1 at a second RARE associated with Rarb (Fig. 5E). Collectively, these data describe a novel mechanism of RA signaling in forebrain-derived neuroepithelial cells that is regulated by GCN5-mediated acetylation of TACC1.

Rescue of (Gcn $\left.5^{\text {hat/hat }}\right)$ phenotype by gestational RA supplementation

Our data supporting a role for RA signaling in the restriction of diencephalic expansion led to the hypothesis that it may be possible to rescue the embryonic defects seen in $\left(G c n 5^{\text {hat } / h a t}\right)$ embryos with RA supplementation. However, this would require some RAR activity to mediate the RA effect. Whereas Rara $^{-/}$; $\mathrm{Rarg}^{-1-}$ embryos display forebrain defects reminiscent of those seen in $\left(G c n 5^{\text {hat } / \text { hat }}\right)$ embryos, Rara $^{-/-} ; \mathrm{Rarb}^{-1-}$ and $\mathrm{Rarg}^{-/-}$; $\mathrm{Rarb}^{-1-}$ embryos do not show forebrain defects (Lohnes et al.,
1994). These data suggest that $\operatorname{RAR} \beta$ may be subject to distinct regulatory mechanisms from those of $\operatorname{RAR} \alpha$ and $\operatorname{RAR} \gamma$. To test this, we first performed coimmunoprecipitation experiments to determine whether RAR $\gamma$ and RAR $\beta$ interact with GCN5 and TACC1 in mouse forebrain tissue at E10.5. As suggested by the genetic data, RAR $\gamma$ coimmunoprecipitated both GCN5 and TACC1, but RAR $\beta$ did not (Fig. 6A). Thus, RAR $\beta$ may act independently of GCN5 and RAR $\beta$ may mediate some of the RA transcriptional response seen even when GCN5 activity is inhibited or absent (Fig. 4D,E). To test these ideas further, we knocked down RAR $\beta$ in NE-4C cells using a combination of two shRNAs (shRarb, Fig. $6 B$, right) and then treated the cells with $100 \mu \mathrm{M}$ MB-3 and increasing concentrations of RA. We then performed qRT-PCR using Foxa1 as a proxy for RA signaling based on our previous experiments (Fig. 4D). Cells treated with shCtrl exhibited increased levels of Foxal expression in response to higher levels of RA, suggesting that increased RA can overcome the RA signaling deficits caused by MB-3-mediated GCN5 inhibition (Fig. $6 B$; MB-3 + shCtrl $0.01 \mu \mathrm{M}$ RA vs $1.0 \mu \mathrm{M}$ RA 95\% CI of 
A

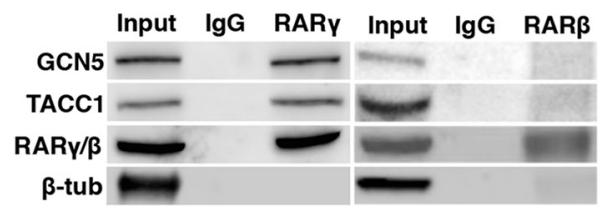

B
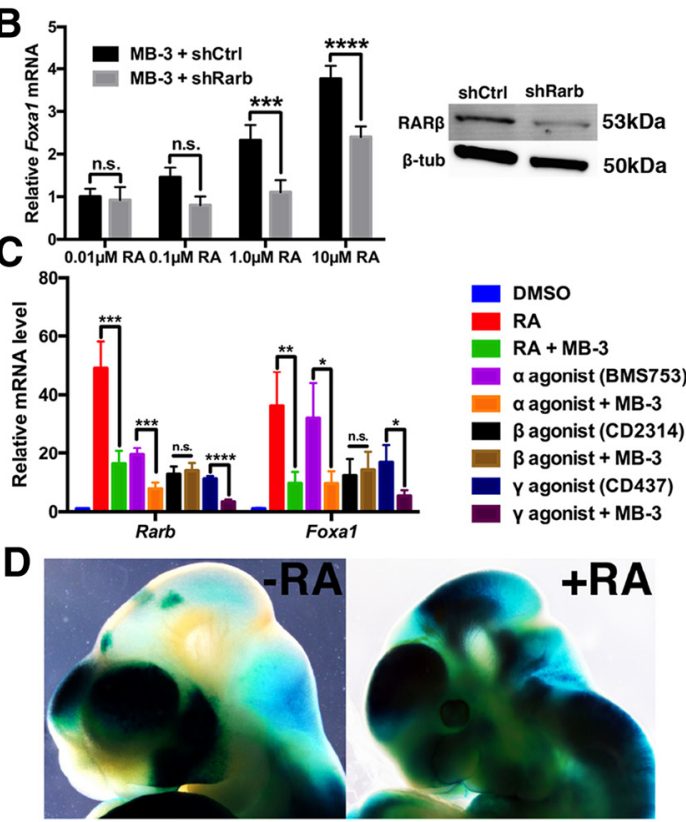

RAREhsplacZ

E

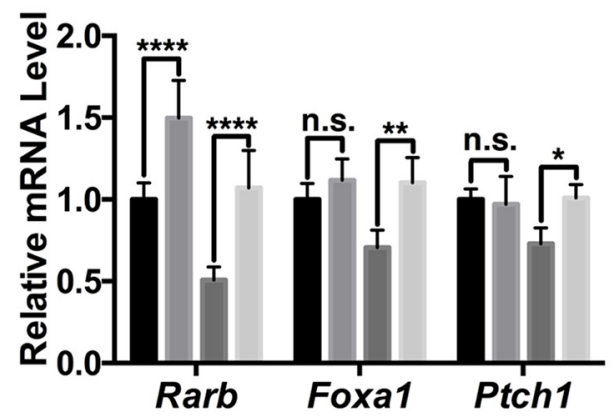

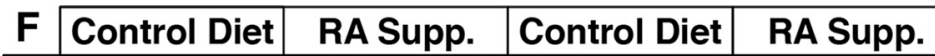

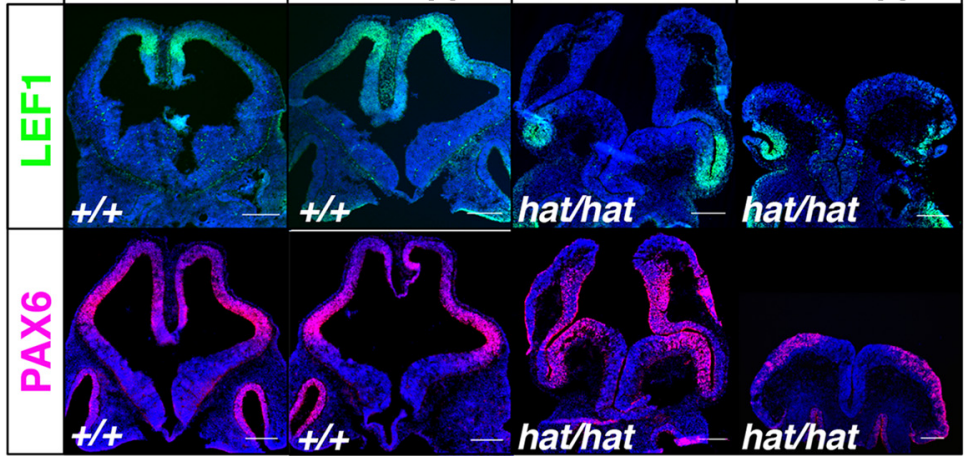

G

H Diencephalic Length
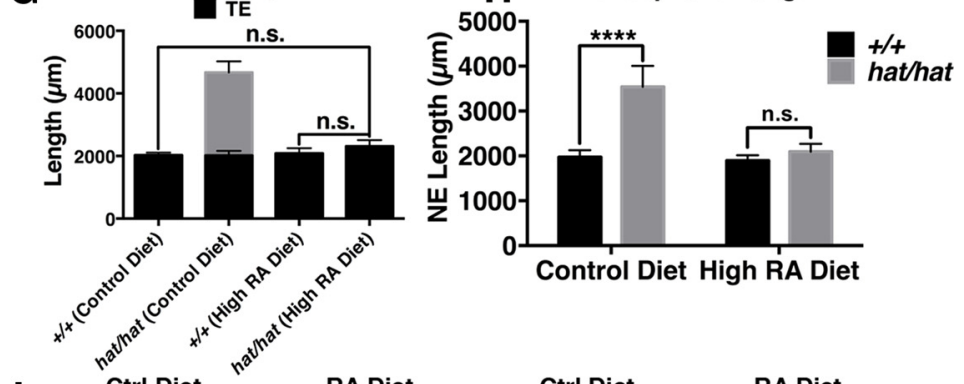
$+/+$
hat/hat

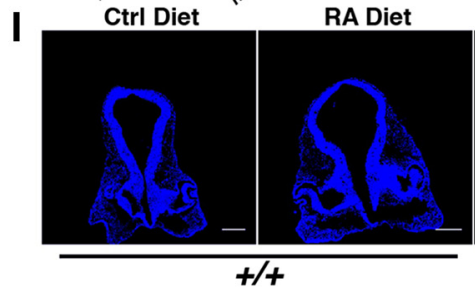

Ctrl Diet

RA Diet

$+/+($ Ctrl Diet $)$

+/+ (High RA Diet)

hat/hat (Ctrl Diet)

hat/hat (High RA Diet)

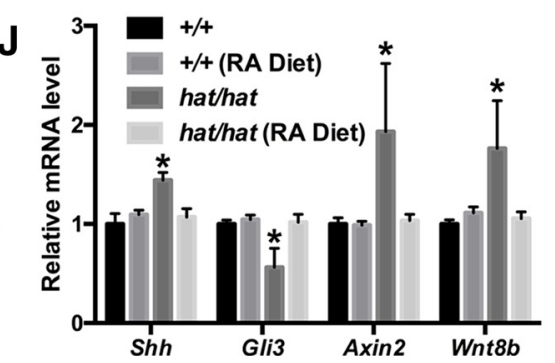

Figure 6. Gestational RA supplementation rescues diencephalic expansion in (Gcn5 ${ }^{\text {hat } / \text { hat }}$ ) embryos. A, Coimmunoprecipitation for RAR $\gamma$ indicates association with both TACC 1 and GCN5 in E10.5 forebrain tissue. Coimmunoprecipitation with anti-RAR $\beta$ antibody shows that RAR $\beta$ does not associate with GCN5 or TACC1 in E10.5 forebrain tissue ( $N=3)$. B, Knockdown of Rarb (shRarb) in NE-4C cells treated with $100 \mu \mathrm{m}$ MB-3 and increasing concentrations of RA followed by qRT-PCR indicates that RAR $\beta$ is required for RA concentration-dependent expression of Foxa 1 when GCN5 activity is lost ( $N=4$ per condition). C, Treatment of NE-4C cells with varying combinations of RA, MB-3, BMS 753 (a selective RAR $\alpha$ agonist), CD 2314 (a selective RAR $\beta$ agonist), and CD 437 (a selective RAR $\gamma$ agonist) for $24 \mathrm{~h}$ indicates that only RAR $\beta$-mediated target gene expression is independent of GCN5 enzymatic activity ( $N=4$ replicates per condition). $\boldsymbol{D}$, $\beta$-Galactosidase staining shows that gestational RA supplementation of RAREhsplacZ mice results in widespread expansion of lacZ expression, a proxy of RA signaling, in the developing brain at E10.5. E, qRT-PCR for Rarb, Foxa1, and Ptch 1 in forebrain tissue collected from wild-type and mutant E10.5 embryos on control and high-RA diet shows a significant upregulation of expression in response to the high-RA diet in mutant embryos to expression levels that are indistinguishable from wild-type levels on control diet ( $N=3$ embryos per genotype per diet). $\boldsymbol{F}$, Dietary RA supplementation starting at gestational age E8.5 prevents diencephalic expansion in E10.5 (Gcn5 ${ }^{\text {hat } / \text { hat }}$ ) embryos but does not rescue NTD. RA supplementation normalizes LEF1 and PAX6 in (Gcn5 ${ }^{\text {hat } / \text { hat }}$ ) embryos such that LEF1 is restricted to the dorsal-most region of the pallium and PAX6 expression begins at the pallial-subpallial border and extends to the dorsal margin of the open neural tissue. These expression patterns are similar to wild-type embryos at this dose of RA that does not disrupt wild-type patterning ( $N \geq 5$ RA treated embryos per genotype). $G$, Measurement of the dorsal-ventral neuroepithelial length in the telencephalic region of wild-type and ( $G \mathrm{Cn} 5^{\text {hat } / \text { hat }}$ ) embryos on control and high-RA diets demonstrates that gestational RA supplementation is sufficient to rescue the overgrowth of the diencephalic

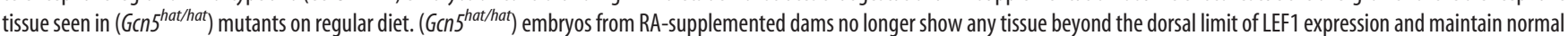
telencephalic length ( $N \geq 4$ per genotype/condition). $\boldsymbol{H}$, Dietary RA supplementation restores the length of the diencephalic neuroepithelium (NE) of (Gcn $5^{\text {hat } / \text { at }}$ ) embryos to normal wild-type length ( $N \geq 5$ animals per genotype). $I$, Nuclear staining of wild-type and ( $G\left(n 5^{\text {hat } / \text { hat }}\right)$ embryos in the region of the diencephalon demonstrates that gestational RA supplementation is capable of rescuing the significant diencephalic expansion observed in (Gcns ${ }^{\text {hat } / \text { hat }}$ ) mutants ( $N \geq 5$ per genotype/condition). Scale bars, $150 \mu \mathrm{m}$.J, Gestational RA supplementation rescues expression of Shh, Gli3, Axin2, and Wnt8b to wild-type levels ( $N=4$ animals per genotype). ${ }^{*} p<0.05 .{ }^{* *} p<0.01 .{ }^{* * *} p<0.001 .{ }^{* * * *} p<0.0001$. n.s., $p>0.05$.

difference -2.087 to -0.5599 , adjusted $p=0.0004$; MB-3 + shCtrl $0.01 \mu \mathrm{M}$ RA vs $10 \mu \mathrm{M}$ RA 95\% CI of difference -3.527 to -2.000 , adjusted $p<0.0001 ; N=3$ wells per condition, two-way ANOVA, Tukey's multiple-comparisons test). Knockdown of
$\operatorname{RAR} \beta$, however, significantly decreased this RA concentrationdependent transcriptional response (Fig. 6B, $1.0 \mu \mathrm{M}$ RA + MB-3 shCtrl vs shRarb 95\% CI of difference 0.4544 to 1.981 , adjusted $p=0.0009 ; 10 \mu \mathrm{M}$ RA + MB-3 shCtrl vs shRarb 95\% CI of 
difference 0.5999 to 2.127 , adjusted $p=0.0003 ; N=3$ wells per condition, two-way ANOVA, Tukey's multiple-comparisons test), indicating that RAR $\beta$ is required for RA-mediated rescue of Foxa1 expression upon inhibition of GCN5 enzymatic activity. We further assayed this by treating NE-4C cells with MB-3 and a combination of selective RAR agonists, which revealed that GCN 5 activity is required for robust $\operatorname{RAR} \alpha$-and $\operatorname{RAR} \gamma$-mediated gene expression, but not $\operatorname{RAR} \beta$ agonist-mediated expression (Fig. $6 C ; N=4$ wells per condition, unpaired $t$ test). Together, these experiments indicate that increasing RA concentrations can overcome the RA signaling deficiency caused by GCN5 inhibition and that this rescue of gene expression requires the activity of $\operatorname{RAR} \beta$.

Rarb is expressed in the forebrain and reduced by $\sim 50 \%$ upon loss of GCN5 activity (Fig. 4D). This led us to test in vivo whether it may be possible to rescue the diencephalic expansion in $\left(G c n 5^{\text {hat } / \text { hat }}\right)$ embryos by increasing RA signaling in the embryo, perhaps through the RAR $\beta$-dependent mechanism described in Figure $6 C$. To test this hypothesis, we performed timed matings of $\mathrm{Gcn}^{\text {hat/+ }}$ animals and administered $175 \mathrm{mg} \mathrm{RA} / \mathrm{kg}$ food to pregnant dams from gestational day 8.5 until embryo harvest at E10.5, followed by analysis for changes in the forebrain phenotype. This diet has been used in published experiments and is sufficient to drive widespread increases in RA signaling in the murine embryo (Fig. 6D) (Harrison-Uy et al., 2013). Wild-type embryos collected from pregnant dams on diet had normal morphology and forebrain patterning (Fig. $6 D, F$ ), suggesting that the level of RA provided to the pregnant dams was within a safe range for the embryos. qRT-PCR using forebrain tissue from E10.5 wild-type and mutant embryos on control and high-RA diet showed significant upregulation of Rarb, Foxa1, and Ptch1 in response to the RA supplementation (Fig. 6E). Importantly, $\left(G c n 5^{\text {hat } / \text { hat }}\right)$ embryos from dams on the high-RA diet displayed levels of these transcripts that were indistinguishable from those in wild-type embryos recovered from dams on control diet ( $N=3$ embryos per genotype per diet, two-way ANOVA, Tukey's multiple-comparisons test, $p>0.05)$. (Gcn $\left.5^{\text {hat } / \text { at }}\right) \mathrm{em}$ bryos from RA-supplemented dams still displayed NTDs, suggesting that the level of RA administered was not sufficient to rescue the NTD or that NTDs occur in $\left(G c n 5^{\text {hat } / \text { hat }}\right)$ embryos due to an RA-independent mechanism. Strikingly, however, gestational RA supplementation was sufficient to rescue the forebrain patterning defects described in Figure 1, as indicated by a reversion of LEF1 and PAX6 staining to their normal, dorsally restricted domains of the neuroepithelium (Fig. $6 F$ ). Importantly, there was no longer ectopic DE tissue observed beyond the dorsal limits of the LEF1 and PAX6 domains (Fig. 6F, G). Despite the persistent open neural tube, measurement of the dorsal-ventral neuroepithelial length in the TE region of the embryos from dams on RA diet revealed a complete rescue of the ectopic DE, with no changes in telencephalic length due to dietary RA supplementation (Fig. $6 \mathrm{G} ; \mathrm{N}=6 \mathrm{Gcn}^{+/+}$control diet, $4 \mathrm{Gcn} 5^{+/+}$high-RA diet, and $4\left(G c n 5^{\text {hat } / h a t}\right)$ high-RA diet, two-way ANOVA, Tukey's multiple-comparisons test, $p>0.05)$. Furthermore, there was no statistically significant difference in length of the diencephalic neuroepithelium in RA-treated (Gcn5 $5^{\text {hat } / \text { hat }}$ ) mutant embryos compared with RA-treated wild-type (Fig. $6 \mathrm{H}, \mathrm{I}$, 95\% CI of difference -659.4 to 263.3 , adjusted $p=0.627$ ), indicating that RA supplementation is capable of rescuing expansion of the diencephalon and restricting the diencephalon to its normal size and domain. Last, in support of this rescue occurring through the signaling pathways assayed in Figure 2, we observed a rescue of the expression levels of Shh, Gli3, Axin2, and Wnt8b in forebrain tissue collected from RA-supplemented ( $G c n 5^{\text {hat }}$ hat $)$ embryos (Fig. 6J, $N=4$ embryos per genotype per diet, two-way ANOVA, Tukey's multiple-comparisons test, $p>0.05$ ). Thus, RA supplementation can rescue the diencephalic expansion caused by loss of GCN5 enzymatic activity through the modulation of normal signaling pathways in the diencephalon.

\section{Discussion}

Our studies identify a novel, non-chromatin-related function of GCN5 that is required to restrict diencephalic expansion during early forebrain development via facilitation of RA signaling. $\left(G c n 5^{\text {hat/hat }}\right)$ embryos, in which GCN5 protein is present but enzymatically inactive, show a significantly expanded diencephalon and compression of the telencephalon. We mechanistically linked this to diencephalon-specific loss of RA signaling that leads to increased WNT activity, decreased Gli3 expression, and increased Shh expression in the diencephalic organizing center known as the ZLI. We then identified a physical interaction between GCN5, RAR $\alpha$, and TACC1 showed that this complex is enriched at certain RARE sites and demonstrated that GCN5 acts to acetylate TACC1, causing dissociation of TACC1 from the complex and target RAREs to promote RA-mediated transcription. Finally, we confirmed the link between GCN5 activity and regulation of RA signaling in vivo by rescuing the diencephalic expansion in mutants with RA supplementation of pregnant dams. Together, these studies indicate that GCN5 is a key integrator of negative regulatory mechanisms of diencephalic growth during early neural development.

Although several previous studies concluded that RA signaling is dispensable for gross morphological development of forebrain structures (Lohnes et al., 1994; Molotkova et al., 2007), our data clearly indicate that RA signaling and GCN5 function is required in the dorsal diencephalon between E9.5 and E10.5 to prevent severe expansion of diencephalic tissue. Rara $^{-1-}$; $\mathrm{Rarg}^{-/-}$embryos at E10.5 show similar forebrain morphology defects as (Gcn5 $\left.5^{\text {hat } / \text { at }}\right)$ embryos, and even though only a small proportion of $\mathrm{Rara}^{-/-}$; $\mathrm{Rarg}^{-/-}$embryos have hindbrain exencephaly, the authors hypothesized that the observed forebrain defects were due to changes in ventricular pressure (Lohnes et al., 1994). Our data, however, strongly support an alternative hypothesis: that the neuroepithelial expansion and telencephalic compression observed in the absence of proper RA signaling in the forebrains of these embryos are due to $\mathrm{SHH}$-induced overgrowth of the diencephalon. The lack of forebrain exencephaly in $\mathrm{Rara}^{-/-} ; \mathrm{Rarg}^{-1-}$ mutants combined with the inability of RA supplementation to rescue the NTDs observed in (Gcn $\left.5^{\text {hat } / \text { hat }}\right)$ embryos suggests that RA signaling is not essential for neural tube closure in the forebrain and that GCN5 controls other non-RA targets during neural tube formation, an area of research for future study. These conclusions are further supported by diencephalic expansion observed in Raldh $3^{-/-}$embryos in the absence of any forebrain neural tube defects (Molotkova et al., 2007).

Specification and patterning of the diencephalon have been extensively studied, but the hierarchy of necessary signaling molecules has remained unresolved. One line of study supports a model whereby WNT signaling inhibits Gli3 expression in the alar plate, rendering this region competent to express $\mathrm{SHH}$ and become the ZLI (Martinez-Ferre et al., 2013). Other studies contest that the master regulator of diencephalic specification is $\mathrm{SHH}$, with GLI3 playing a necessary role in shaping the ZLI (Rash and Grove, 2011). Meanwhile, it has remained unclear what role, if any, RA signaling plays. In chick and quail, the RA-synthesizing enzyme CYP1B1 is expressed in the dorsal-most region of the diencephalon where $\mathrm{SHH}$ expression is 


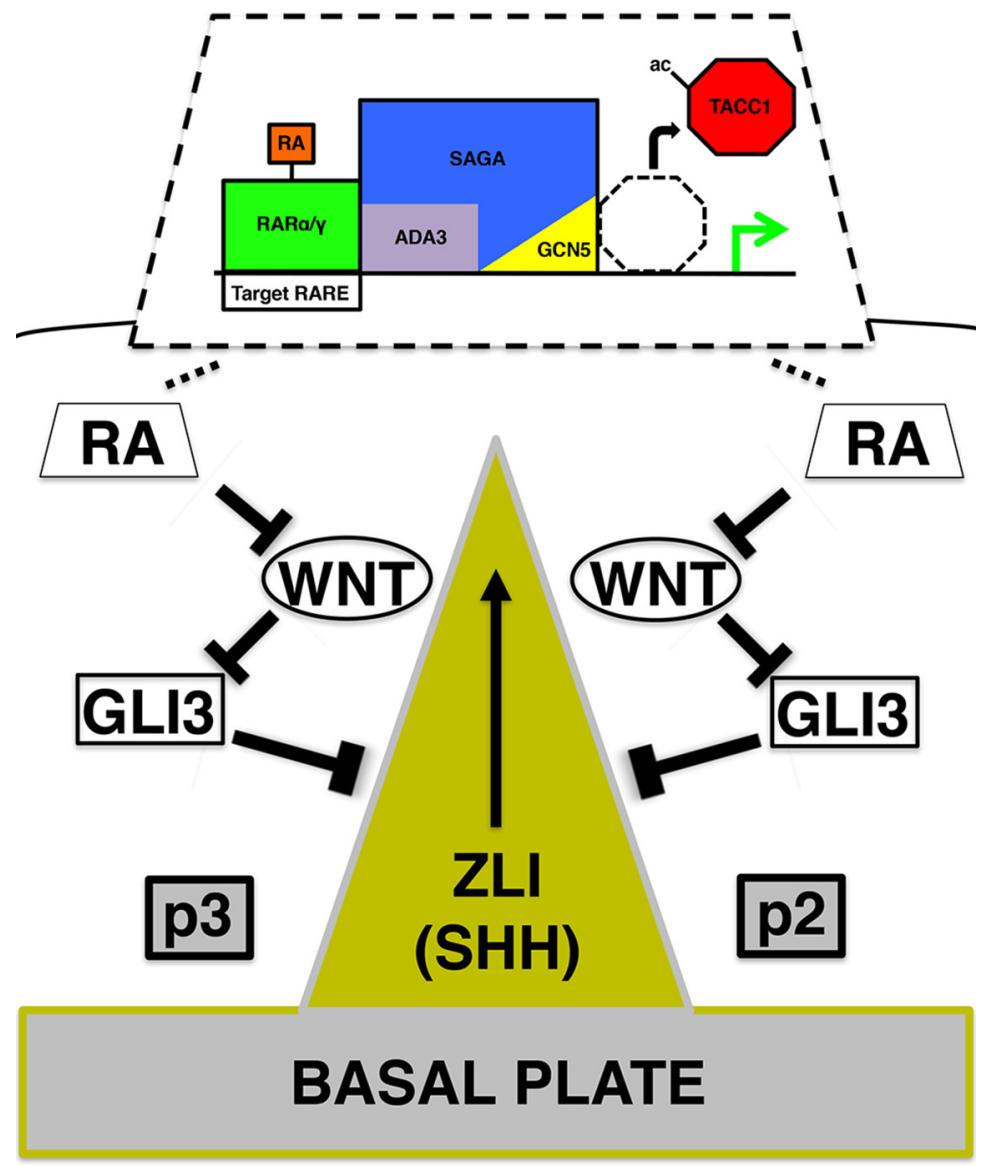

Figure 7. A proposed model for GCN5-mediated regulation of diencephalic size. In the absence of RA, a poised transcriptional complex containing RAR $\alpha$ or RAR $\gamma$, GCN5/SAGA, and TACC 1 localizes to the RAREs of some RA-responsive genes, with TACC1 acting as a negative regulator of transcription in the absence of ligand. Upon RA binding to the RAR, GCN5-mediated acetylation of TACC 1 causes dissociation of TACC 1 from the complex, allowing transcription of downstream RA target genes. RA signaling in the dorsal p3 and p2 domains of the developing diencephalon inhibits WNT signaling, which is a positive regulator of Shh expression in the alar plate through its inhibition of Gli3 expression. Loss of GCN5 activity results in decreased RA signaling, which causes widespread upregulation of WNT and SHH through increased repression of Gli3 expression and expansion of the ZLI.

suppressed (Chambers et al., 2007; Guinazu et al., 2007). Diencephalic explant experiments demonstrated that regions flanking the ZLI are required to shape the domain of Shh expression (Guinazu et al., 2007), and these regions correspond to the diencephalic expression domains of the RA synthesizing enzyme RALDH3 (Molotkova et al., 2007). Our finding that the regions flanking the ZLI are sites of active RA signaling (Fig. 4C) and that the absence of this RA signaling in $\left(G c n 5^{\text {hat } / \text { hat }}\right)$ embryos correlates with increased WNT signaling, decreased Gli3 expression, and increased Shh expression supports a key role of RA in shaping the developing diencephalon. RA can directly inhibit WNT signaling in multiple contexts (Easwaran et al., 1999; Shah et al., 2003; S. Li et al., 2008), and our data suggest that RA is the most upstream factor in determining diencephalic size through inhibition of WNT. Supporting this hypothesis, qRT-PCR experiments using RNA from E9.5 embryos that do not yet display diencephalic expansion revealed significant dysregulation of WNT pathway genes and Rarb, but not Gli3 and Shh, suggesting that GCN5 and RA lie upstream of WNT, which then acts to regulate diencephalic $\mathrm{SHH}$ signaling and diencephalic proliferation. Together, our work supports a model in which GCN5, RA, WNT, GLI3, and SHH are tightly interrelated and function together to create a fine balance that controls diencephalic size and border specification between forebrain structures.

GCN5 has been implicated in RA signaling, although its hypoth- esized role has been restricted to promotion of transcription through histone acetylation (Brown et al., 2003). While the direct association of SAGA complex member ADA3 with RAR $\alpha$ supports this hypothesis (C. W. Li et al., 2010), functional studies investigating the direct role of GCN5-mediated histone acetylation in RA signaling are lacking. Furthermore, recent studies indicate that GCN5 elicits numerous tissue-specific functions through direct acetylation of nonhistone proteins, including $\alpha$-tubulin (Conacci-Sorrell et al., 2010; L. Li et al., 2015), transcription factors (Jacob et al., 2001; Zelin et al., 2012), and the transcriptional coactivator PGC1 $\alpha$ (Sakai et al., 2012). Our findings identify a new tissuespecific regulatory role for GCN5-catalyzed acetylation that involves the substrate TACC1, which we find acts as a negative regulator of RA signaling in the diencephalon (model in Fig. 7). Although TACC1 interaction with hormone receptors has been described and TACC1 nuclear localization highlighted (Lauffart et al., 2006; Vettaikkorumakankauv et al., 2008; Guyot et al., 2010), previous studies of the TACC family of proteins have only focused on their regulation of the cytoskeleton through interactions with Ch-TOG and Aurora A/B/C (Peset and Vernos, 2008; Gabillard et al., 2011). Interestingly, the same domain of TACC1 can be bound by either GCN5 or Ch-TOG, suggesting that this domain's function is dependent upon subcellular location. The TACC family of proteins can stabilize microtubules during mitosis through recruitment of proteins to centrosomes, suggesting that TACC1 functions as a scaffold for the assembly of protein complexes (Peset and Vernos, 2008; Thakur et al., 2014). Interestingly, previous work showing that knockdown of Taccl results in decreased RA target gene expression (Guyot et al., 2010) suggests that TACC1 may serve a dual role as both an adaptor protein necessary for assembly of "poised" RA regulatory complexes on RAREs and a transcriptional repressor that blocks transcription in the absence of ligand (RA).

Our data indicate that $\operatorname{RAR} \alpha / \operatorname{RAR} \gamma, \mathrm{GCN} 5$, and TACC1 are poised on RAREs in neuroepithelial cells before ligand binding. Upon RA binding, GCN5-mediated acetylation of TACC1 causes dissociation of TACC1 from the RA transcriptional complex, allowing rapid and precise transcriptional activation of RAresponsive genes in the diencephalon. Moreover, GCN5 could play multiple roles in RA signaling: functioning both to remove TACC1-mediated transcriptional repression and to increase transcriptional efficiency via histone acetylation and subsequent opening of the local chromatin structure. The studies of ADA3 interaction with RAR $\alpha$ show interaction in the absence of RA that is greatly enhanced by ligand binding (C. W. Li et al., 2010), similar to the results observed in our ChIP assays (Fig. 5E). These results indicate that GCN5-mediated acetylation of TACC1 could alleviate transcriptional repression while allowing recruitment and/or activation of other SAGA complex members as a means of 
driving RA target gene transcription through chromatin modification and recruitment of the transcriptional machinery.

The mechanistic work and rescue experiments that are described in Figures 5 and 6 illustrate several key points about RA signaling and its role in forebrain development. First, they support previously published results indicating that RA signaling in the developing forebrain is highly redundant. While several lines of research have argued that RA signaling is largely dispensable for gross development of forebrain structures, our studies support previous data suggesting that this is largely due to compensatory mechanisms between the RAR family members and that disruption of the activities of multiple RARs due to loss of GCN5 function causes severe neurodevelopmental defects. Normally, GCN5 does not function through $\operatorname{RAR} \beta$ and $\operatorname{RAR} \beta$ is typically expressed at low levels in the developing diencephalon (Yamagata et al., 1994). However, analysis of embryos whose mothers were treated with a high-RA diet indicates significant upregulation of Rarb expression in forebrain tissue and widespread upregulation of RA signaling throughout the embryo. This suggests that the phenotypic rescue observed in response to the high-RA diet in (Gcn $\left.5^{\text {hat/hat }}\right)$ embryos results from increased RA signaling in the diencephalon through a feedforward mechanism of Rarb expression and subsequent RAR $\beta$-mediated transcriptional activation of essential diencephalic RA target genes. We cannot exclude that this rescue occurs through different targets than those normally regulated by GCN5-TACC1-RAR $\alpha$ / $\operatorname{RAR} \gamma$ complexes or $\operatorname{RAR} \beta$, but the normalization of forebrain patterning and growth, as well as the rescue of Shh, Gli3, Axin2, and $W n t 8 b$ transcript levels suggests the involvement of at least some of the normal regulators of diencephalic development. Future studies using a combination of ChIP-seq and RNA-seq approaches in wild-type and mutant embryos on both control and RA-supplemented diets are needed to elucidate these important targets of RA signaling in the diencephalon. Together, our findings describe a novel function of the acetyltransferase GCN5 and a novel regulatory mechanism of RA signaling that are both critical for gross morphological development of the diencephalon.

\section{References}

Al Tanoury Z, Piskunov A, Rochette-Egly C (2013) Vitamin A and retinoid signaling: genomic and nongenomic effects. J Lipid Res 54:1761-1775. CrossRef Medline

Balmer JE, Blomhoff R (2002) Gene expression regulation by retinoic acid. J Lipid Res 43:1773-1808. CrossRef Medline

Besnard V, Wert SE, Hull WM, Whitsett JA (2004) Immunohistochemical localization of Foxa1 and Foxa2 in mouse embryos and adult tissues. Gene Expr Patterns 5:193-208. CrossRef Medline

Biel M, Kretsovali A, Karatzali E, Papamatheakis J, Giannis A (2004) Design, synthesis, and biological evaluation of a small-molecule inhibitor of the histone acetyltransferase Gcn5. Angew Chem Int Ed 43:3974-3976. CrossRef Medline

Brand M, Yamamoto K, Staub A, Tora L (1999) Identification of TATAbinding protein-free TAFII-containing complex subunits suggests a role in nucleosome acetylation and signal transduction. J Biol Chem 274: 18285-18289. CrossRef Medline

Brown K, Chen Y, Underhill TM, Mymryk JS, Torchia J (2003) The coactivator $\mathrm{p} / \mathrm{CIP} / \mathrm{SRC}-3$ facilitates retinoic acid receptor signaling via recruitment of GCN5. J Biol Chem 278:39402-39412. CrossRef Medline

Bu P, Evrard YA, Lozano G, Dent SY (2007) Loss of Gcn5 acetyltransferase activity leads to neural tube closure defects and exencephaly in mouse embryos. Mol Cell Biol 27:3405-3416. CrossRef Medline

Busch AM, Galimberti F, Nehls KE, Roengvoraphoj M, Sekula D, Li B, Guo Y, Direnzo J, Fiering SN, Spinella MJ, Robbins DJ, Memoli VA, Freemantle SJ, Dmitrovsky E (2014) All-trans-retinoic acid antagonizes the Hedgehog pathway by inducing patched. Cancer Biol Ther 15:463-472. CrossRef Medline

Candau R, Moore PA, Wang L, Barlev N, Ying CY, Rosen CA, Berger SL
(1996) Identification of human proteins functionally conserved with the yeast putative adaptors ADA2 and GCN5. Mol Cell Biol 16:593-602. CrossRef Medline

Carradori S, Secci D, Mai A (2011) Epigenetic modulation of PGC- $1 \alpha$ activity by GCN5 inhibitors: WO2010007085. Expert Opin Ther Pat 21: 1651-1656. CrossRef Medline

Chakravarti D, LaMorte VJ, Nelson MC, Nakajima T, Schulman IG, Juguilon H, Montminy M, Evans RM (1996) Role of CBP/P300 in nuclear receptor signalling. Nature 383:99-103. CrossRef Medline

Chambers D, Wilson L, Maden M, Lumsden A (2007) RALDHindependent generation of retinoic acid during vertebrate embryogenesis by CYP1B1. Development 134:1369-1383. CrossRef Medline

Chatterjee M, Guo Q, Weber S, Scholpp S, Li JY (2014) Pax6 regulates the formation of the habenular nuclei by controlling the temporospatial expression of Shh in the diencephalon in vertebrates. BMC Biol 12:13. CrossRef Medline

Chen JF, Zhang Y, Wilde J, Hansen KC, Lai F, Niswander L (2014) Microcephaly disease gene Wdr62 regulates mitotic progression of embryonic neural stem cells and brain size. Nat Commun 5:3885. CrossRef Medline

Chen J, Lai F, Niswander L (2012) The ubiquitin ligase mLin41 temporally promotes neural progenitor cell maintenance through FGF signaling. Genes Dev 26:803-815. CrossRef Medline

Choi J, Park S, Sockanathan S (2014) Activated retinoid receptors are required for the migration and fate maintenance of subsets of cortical neurons. Development 141:1151-1160. CrossRef Medline

Conacci-Sorrell M, Ngouenet C, Eisenman RN (2010) Myc-nick: a cytoplasmic cleavage product of Myc that promotes alpha-tubulin acetylation and cell differentiation. Cell 142:480-493. CrossRef Medline

Easwaran V, Pishvaian M, Salimuddin, Byers S (1999) Cross-regulation of beta-catenin-LEF/TCF and retinoid signaling pathways. Curr Biol 9:1415-1418. CrossRef Medline

Epstein DJ (2012) Regulation of thalamic development by sonic hedgehog. Front Neurosci 6:1-6. CrossRef Medline

Epstein DJ, Martinu L, Michaud JL, Losos KM, Fan C, Joyner AL (2000) Members of the bHLH-PAS family regulate Shh transcription in forebrain regions of the mouse CNS. Development 127:4701-4709. Medline

Gabillard JC, Ulisse S, Baldini E, Sorrenti S, Cremet JY, Coccaro C, Prigent C, D'Armiento M, Arlot-Bonnemains Y (2011) Aurora-C interacts with and phosphorylates the transforming acidic coiled-coil 1 protein. Biochem Biophys Res Commun 408:647-653. CrossRef Medline

Gangisetty O, Lauffart B, Sondarva GV, Chelsea DM, Still IH (2004) The transforming acidic coiled coil proteins interact with nuclear histone acetyltransferases. Oncogene 23:2559-2563. CrossRef Medline

Georgakopoulos T, Thireos G (1992) Two distinct yeast transcriptional activators require the function of the GCN5 protein to promote normal levels of transcription. EMBO J 11:4145-4152. Medline

Gilmore EC, Walsh CA (2013) Genetic causes of microcephaly and lessons for neuronal development. Wiley Interdiscip Rev Dev Biol 2:461-478. CrossRef Medline

Grant PA, Eberharter A, John S, Cook RG, Turner BM, Workman JL (1999) Expanded lysine acetylation specificity of $\mathrm{Gcn} 5$ in native complexes. J Biol Chem 274:5895-5900. CrossRef Medline

Guinazu MF, Chambers D, Lumsden A, Kiecker C (2007) Tissue interactions in the developing chick diencephalon. Neural Dev 2:25. CrossRef Medline

Gupta S, Sen J (2015) Retinoic acid signaling regulates development of the dorsal forebrain midline and the choroid plexus in the chick. Development 142:1293-1298. CrossRef Medline

Guyot R, Vincent S, Bertin J, Samarut J, Ravel-Chapuis P (2010) The transforming acidic coiled coil (TACC1) protein modulates the transcriptional activity of the nuclear receptors TR and RAR. BMC Mol Biol 11:3. CrossRef Medline

Haddad-Tóvolli R, Heide M, Zhou X, Blaess S, Alvarez-Bolado G (2012) Mouse thalamic differentiation: gli-dependent pattern and gliindependent prepattern. Front Neurosci 6:27. CrossRef Medline

Harmacek L, Watkins-Chow DE, Chen J, Jones KL, Pavan WJ, Salbaum JM, Niswander L (2014) A unique missense allele of BAF155, a core BAF chromatin remodeling complex protein, causes neural tube closure defects in mice. Dev Neurobiol 74:483-497. CrossRef Medline

Harrison-Uy SJ, Siegenthaler JA, Faedo A, Rubenstein JL, Pleasure SJ (2013) CoupTFI interacts with retinoic acid signaling during cortical development. PLoS One 8:e58219. CrossRef Medline 
Himmelstein DS, Bi C, Clark BS, Bai B, Kohtz JD (2010) Balanced Shh signaling is required for proper formation and maintenance of dorsal telencephalic midline structures. BMC Dev Biol 10:118. CrossRef Medline

Holmes G, Niswander L (2001) Expression of slit-2 and slit-3 during chick development. Dev Dyn 222:301-307. Medline

Hou N, Ren L, Gong M, Bi Y, Gu Y, Dong Z, Liu Y, Chen J, Li T (2015) Vitamin A deficiency impairs spatial learning and memory: the mechanism of abnormal CBP-dependent histone acetylation regulated by retinoic acid receptor alpha. Mol Neurobiol 51:633-647. CrossRef Medline

Jacob AL, Lund J, Martinez P, Hedin L (2001) Acetylation of steroidogenic factor 1 protein regulates its transcriptional activity and recruits the coactivator GCN5. J Biol Chem 276:37659-37664. CrossRef Medline

Jacob A, Budhiraja S, Reichel RR (1999) The HNF-3 $\alpha$ transcription factor is a primary target for retinoic acid action. Exp Cell Res 250:1-9. CrossRef Medline

Jho EH, Zhang T, Domon C, Joo CK, Freund JN, Costantini F (2002) Wnt/ beta-catenin/Tcf signaling induces the transcription of Axin2, a negative regulator of the signaling pathway. Mol Cell Biol 22:1172-1183. CrossRef Medline

Lalevée S, Anno YN, Chatagnon A, Samarut E, Poch O, Laudet V, Benoit G, Lecompte O, Rochette-Egly C (2011) Genome-wide in silico identification of new conserved and functional retinoic acid receptor response elements (direct repeats separated by 5 bp). J Biol Chem 286:3332233334. CrossRef Medline

Lancaster MA, Renner M, Martin CA, Wenzel D, Bicknell LS, Hurles ME, Homfray T, Penninger JM, Jackson AP, Knoblich JA (2013) Cerebral organoids model human brain development and microcephaly. Nature 501:373-379. CrossRef Medline

Lauffart B, Dimatteo A, Vaughan MM, Cincotta MA, Black JD, Still IH (2006) Temporal and spatial expression of TACC1 in the mouse and human. Dev Dyn 235:1638-1647. CrossRef Medline

Lerin C, Rodgers JT, Kalume DE, Kim SH, Pandey A, Puigserver P (2006) GCN5 acetyltransferase complex controls glucose metabolism through transcriptional repression of PGC- $1 \alpha$. Cell Metab 3:429-438. CrossRef Medline

Li CW, Ai N, Dinh GK, Welsh WJ, Chen JD (2010) Human ADA3 regulates RARalpha transcriptional activity through direct contact between LxxLL motifs and the receptor coactivator pocket. Nucleic Acids Res 38:52915303. CrossRef Medline

Li L, Liu B, Zhang X, Ye L (2015) The oncoprotein HBXIP promotes migration of breast cancer cells via GCN5-mediated microtubule acetylation. Biochem Biophys Res Commun 458:720-725. CrossRef Medline

Li S, Lou X, Wang J, Liu B, Ma L, Su Z, Ding X (2008) Retinoid signaling can repress blastula Wnt signaling and impair dorsal development in Xenopus embryo. Differentiation 76:897-907. CrossRef Medline

Lohnes D, Mark M, Mendelsohn C, Dollé P, Dierich A, Gorry P, Gansmuller A, Chambon P (1994) Function of the retinoic acid receptors (RARs) during development: I. Craniofacial and skeletal abnormalities in RAR double mutants. Development 120:2723-2748. Medline

Maden M (2007) Retinoic acid in the development, regeneration and maintenance of the nervous system. Nat Rev Neurosci 8:755-765. CrossRef Medline

Martinez-Ferre A, Navarro-Garberi M, Bueno C, Martinez S (2013) Wnt signal specifies the intrathalamic limit and its organizer properties by regulating Shh induction in the alar plate. J Neurosci 33:3967-3980. CrossRef Medline

Mavromatakis YE, Lin W, Metzakopian E, Ferri AL, Yan CH, Sasaki H, Whisett J, Ang SL (2011) Foxa1 and Foxa2 positively and negatively regulate Shh signalling to specify ventral midbrain progenitor identity. Mech Dev 128:90-103. CrossRef Medline

Merrill RA, Ahrens JM, Kaiser ME, Federhart KS, Poon VY, Clagett-Dame M (2004) All-trans retinoic acid-responsive genes identified in the human SH-SY5Y neuroblastoma cell line and their regulated expression in the nervous system of early embryos. Biol Chem 385:605-614. CrossRef Medline

Mirzaa GM, Rivière JB, Dobyns WB (2013) Megalencephaly syndromes and activating mutations in the PI3K-AKT pathway: MPPH and MCAP. Am J Med Genet C Semin Med Genet 163C:122-130. CrossRef Medline

Molotkova N, Molotkov A, Duester G (2007) Role of retinoic acid during forebrain development begins late when Raldh3 generates retinoic acid in the ventral subventricular zone. Dev Biol 303:601-610. CrossRef Medline
Park UH, Kim EJ, Um SJ (2010) A novel cytoplasmic adaptor for retinoic acid receptor (RAR) and thyroid receptor functions as a derepressor of RAR in the absence of retinoic acid. J Biol Chem 285:34269-34278. CrossRef Medline

Peset I, Vernos I (2008) The TACC proteins: TACC-ling microtubule dynamics and centrosome function. Trends Cell Biol 18:379-388. CrossRef Medline

Rash BG, Grove EA (2011) Shh and Gli3 regulate formation of the telencephalic-diencephalic junction and suppress an isthmus-like signaling source in the forebrain. Dev Biol 359:242-250. CrossRef Medline

Rossant J, Zirngibl R, Cado D, Shago M, Giguère V (1991) Expression of a retinoic acid response element-hsplacZ transgene defines specific domains of transcriptional activity during mouse embryogenesis. Genes Dev 5:1333-1344. CrossRef Medline

Sakai M, Matsumoto M, Tujimura T, Yongheng C, Noguchi T, Inagaki K, Inoue H, Hosooka T, Takazawa K, Kido Y, Yasuda K, Hiramatsu R, Matsuki Y, Kasuga M (2012) CITED2 links hormonal signaling to PGC-1a acetylation in the regulation of gluconeogenesis. Nat Med 18:612-617. CrossRef Medline

Schindelin J, Arganda-Carreras I, Frise E, Kaynig V, Longair M, Pietzsch T, Preibisch S, Rueden C, Saalfeld S, Schmid B, Tinevez J-Y, White DJ, Hartenstein V, Eliceiri K, Tomancak P, Cardona A (2012) Fiji: an opensource platform for biological-image analysis. Nat Meth 9:676-682. CrossRef Medline

Schlett K, Madarász E (1997) Retinoic acid induced neural differentiation in a neuroectodermal cell line immortalized by p53 deficiency. J Neurosci Res 47:405-415. CrossRef Medline

Scholpp S, Lumsden A (2010) Building a bridal chamber: development of the thalamus. Trends Neurosci 33:373-380. CrossRef Medline

Shah S, Hecht A, Pestell R, Byers SW (2003) Trans-repression of $\beta$-catenin activity by nuclear receptors. J Biol Chem 278:48137-48145. CrossRef Medline

Siegenthaler JA, Ashique AM, Zarbalis K, Patterson KP, Hecht JH, Kane MA, Folias AE, Choe Y, May SR, Kume T, Napoli JL, Peterson AS, Pleasure SJ (2009) Retinoic acid from the meninges regulates cortical neuron generation. Cell 139:597-609. CrossRef Medline

Thakur HC, Singh M, Nagel-Steger L, Kremer J, Prumbaum D, Fansa EK, Ezzahoini H, Nouri K, Gremer L, Abts A, Schmitt L, Raunser S, Ahmadian MR, Piekorz RP (2014) The centrosomal adaptor TACC3 and the microtubule polymerase chTOG interact via defined C-terminal subdomains in an Aurora-A kinase independent manner. J Biol Chem 289: 74-88. CrossRef Medline

Vettaikkorumakankauv A, Gangisetty O, Cincotta MA, Hawthorne LA, Cowell JK, Still IH (2008) The TACC proteins are coregulators of the Retinoid X Receptor $\beta$. Cancer Ther 6:805-816.

Vieira C, Martinez S (2006) Sonic hedgehog from the basal plate and the zona limitans intrathalamica exhibits differential activity on diencephalic molecular regionalization and nuclear structure. Neuroscience 143:129_ 140. CrossRef Medline

Vue TY, Bluske K, Alishahi A, Yang LL, Koyano-Nakagawa N, Novitch B, Nakagawa Y (2009) Sonic Hedgehog signaling controls thalamic progenitor identity and nuclei specification in mice. J Neurosci 29:44844497. CrossRef Medline

Xu W, Edmondson DG, Evrard YA, Wakamiya M, Behringer RR, Roth SY (2000) Loss of Gcn512 leads to increased apoptosis and mesodermal defects during mouse development. Nat Genet 26:229-232. CrossRef Medline

Yamagata T, Momoi MY, Yanagisawa M, Kumagai H, Yamakado M, Momoi $\mathrm{T}$ (1994) Changes of the expression and distribution of retinoic acid receptors during neurogenesis in mouse embryos. Brain Res Dev Brain Res 77:163-176. CrossRef Medline

Zelin E, Zhang Y, Toogun OA, Zhong S, Freeman BC (2012) The p23 molecular chaperone and GCN5 acetylase jointly modulate protein-DNA dynamics and open chromatin status. Mol Cell 48:459-470. CrossRef Medline

Zeng H, Hoover AN, Liu A (2010) PCP effector gene Inturned is an important regulator of cilia formation and embryonic development in mammals. Dev Biol 339:418-428. CrossRef Medline

Zohn IE, Anderson KV, Niswander L (2005) Using genomewide mutagenesis screens to identify the genes required for neural tube closure in the mouse. Birth Defects Res Part A Clin Mol Teratol 73:583-590. CrossRef Medline 